\title{
An efficient MIMO scheme with signal space diversity for future mobile communications
}

\author{
Zhanji $\mathrm{Wu}^{* \dagger}$ and Xiang Gao ${ }^{\dagger}$
}

\begin{abstract}
An efficient wireless transmission scheme with the signal space diversity (SSD) is proposed to improve the performance of multiple-input multiple-output (MIMO) systems in fading channels. By introducing the rotated modulation and space-time component interleaver, the proposed scheme jointly optimizes channel coding, modulation, and MIMO and can improve the link reliability and energy efficiency. An optimum spatial component interleaver is proposed to maximize the MIMO achievable rate. Based on the average mutual information (AMI)-maximization criterion, the optimal rotation angles of real-valued signal and complex-valued QAM signal are investigated for the MIMO scheme. For the iterative demapping and decoding (ID) scheme, a simple genetic algorithm (GA) to search binary convolution code (BCC) is also put forward to match the rotated modulation. Simulation results show that the optimized BCC-coded MIMO scheme with SSD-ID outperforms the turbo-coded MIMO scheme with bit-interleaved coded modulation (BICM)-ID by $1.4 \mathrm{~dB}$ signal-to-noise ratio (SNR) gain, while the new scheme has much lower complexity. So, the proposed scheme is simple, efficient, and promising for future wireless communication systems.
\end{abstract}

Keywords: Quadrature amplitude modulation (QAM); Signal space diversity (SSD); Multiple-input multiple-output (MIMO); Iterative demodulation and decoding (ID); Extrinsic information transfer chart (EXIT)

\section{Introduction}

Wireless communications have made a great progress in the recent few years. By introducing more advanced technology, $5 \mathrm{G}$ will provide higher spectral efficiency, more spectrum resources, and more reliability to meet the growing demand for mobile traffic [1].

Bit-interleaved coded modulation (BICM) is a bandwidth-efficient coded modulation scheme which increases the time diversity in fading channels [2,3]. For its iterative version, BICM with iterative demapping and decoding (BICM-ID), the extrinsic information is transferred between the channel decoder and the soft-insoft-out demapper, which is like the serial turbo decoder. Multiple-input multiple-output (MIMO) scheme is the extension of the coding theory on the space domain, so it is also named space-time coding (STC) [4]. Foschini proposed a layered space-time (LST) architecture to process multidimensional signals in the space domain [5]. The BICM-LST is a conventional spectral-efficient spatial

\footnotetext{
*Correspondence: wuzhanji@bupt.edu.cn

${ }^{\dagger}$ Equal contributors

School of Telecommunication Engineering, Beijing University of Posts and

Telecommunications, Xitucheng Road 10, Beijing, China
}

multiplexing technology to deal with MIMO fading channels, and the BICM-threaded layered space-time (TLST) with a cyclic-shift spiral spatial interleaver is regarded as the most efficient method, because the cyclic-shift spatial interleaver introduces effective space diversity for the codeword on each layer [6]. In general, the BICMLST can be viewed as the serial concatenation of the channel coding, modulation, and spatial layered multiplexing. Because BICM-LST exhibits a robust diversity performance on fading channels, it is widely deployed by wireless communication standards.

As for the bandwidth-efficient quadrature amplitude modulation (QAM), uncoded rotated multidimensional modulation schemes over independent Rayleigh fading channels were studied in [7] for the single-input singleoutput (SISO) scheme. Different from the other wellknown diversity (time, frequency, code, space), it has an intrinsic modulation diversity, which is named signal space diversity (SSD). Through the combination of constellation rotation and component interleaver, the schemes can achieve very high modulation diversity, and the error performance over fading channels can approach that on the additive white Gaussian noise (AWGN)

\section{是 Springer}


channels. SSD schemes for SISO system have been extensively researched. In [8], SSD is introduced to the BICM by the means of modifications to the QAM constellation mapper and demapper so as to improve the BICM performance of QAM constellations for broadcasting applications. In [9], a LDPC-coded SSD scheme for multi-level modulation was presented. N.F. Kiyani and J.H. Weber studied the rotated-MPSK SISO BICM-ID system [10,12], which focused on two-dimensional multiphase shift keying (MPSK) scheme. In [11], the performance analysis of BICM-ID with SSD in fading channels is presented. In [13], the extension of BICM-SSD schemes with a non-binary code was proposed. We also proposed coded orthogonal frequency division multiplexing (OFDM) systems with SSD in $[14,15]$. In [16], the schemes combining SSD with SISO-coded BICM and BICM-ID systems were investigated. It provided a new criterion for determining the optimal rotation angle by maximizing the average mutual information (AMI). For the optimization of BICM-ID system, it proved that SSD can mitigate the different-slope problem of the demapper's extrinsic information transfer (EXIT) curve under different channels. However, finding well-matched channel codes for given labeling in BICM-ID system with SSD is still a big challenge.

The combination of signal rotation and space-time coding in MIMO system can effectively improve the diversity gain. In order to achieve full diversity, the quasi-orthogonal space time block codes (QOSTBC) with rotating the constellations of half of the complex symbols has been widely discussed in [17-20]. Some specific optimal rotation angles and corresponding optimization criterions for QAM and phase shift keying (PSK) constellations are provided. A rotation-based method that aims at maximizing the minimum distance in the space-time constellation is proposed in [17]. The proposed scheme shows good improvement of the codes compared to their non-rotated counterparts. In [18], the authors considered the design of rotated QOSTBC for the MISO system. The code designs are based mainly on the rank and the determinant criteria, and the optimal rotation angle $\pi / 6$ can provide full diversity and the optimal coding gain. In [19], the authors proposed to design the signal constellations properly to ensure that the resulting quasi-orthogonal STBCs can guarantee to achieve the full diversity. The optimal rotation angles are determined by maximizing the diversity product. A novel method to exactly derive the coding gain of QSTBC as a function of the rotation angle and the minimum Euclidean distance of twodimensional constellations is proposed in [20]. A coded MIMO scheme for block-fading channels was proposed in [21], which consists of a channel code and a spacetime code. The space-time code is designed based on SSD technique, which allows full spatial multiplexing MIMO transmission and achieves full space diversity. In [22], the uncoded SSD scheme was extended to V-BLAST MIMO systems in order to achieve the maximum diversity gain without additional power or bandwidth consumption. An improved turbo-coded SSD scheme was proposed for MIMO-OFDM BICM system in [23], and the linear minimum mean square error (LMMSE) equalization is utilized for the non-ID MIMO detection. In general, the research of SSD technique in coded MIMO systems is still on the original stage. There are still many open problems. For instance, the optimal rotation angles in current research mainly depend on the maximum product distance introduced in [7]. Unfortunately, this criterion is only valid for the SISO system in high signal-to-noise ratio (SNR) region. As for the coded MIMO scheme, when powerful forward error-correction codes (FECs) are considered, actual SNR can be quite low. Hence, the angle values applied to uncoded SISO system do not lead to the best error performance for the coded modulation MIMO scheme. What is more, current research works mainly focus on local optimizations. For example, most proposed MIMO systems with SSD are only an extension of SISO-SSD system, and all are based on the conventional non-precoding transmitter. The channel coding, QAM modulation, and STC are independent with each other, which is just a straightforward serial concatenation. Hence, the performance of the BICM-LST is still rather far away from the MIMO fading channel capacity. For example, a near-capacity BICM-LST scheme was proposed in [24], which allows the iterative processing of the list sphere detection (LSD) and turbo decoding, but simulation results indicate that the gaps to the MIMO capacity are still more than $2 \mathrm{~dB}$. As each individual optimization becomes mature so far, from the philosophy, it is high time to optimize these key technical elements jointly so as to improve the overall performance.

An improved coded MIMO system based on SSD is proposed for the jointly optimization of constellation rotation angle, spatial component interleaver, and the matching of channel coding and labeling, which is named joint coding and modulation diversity (JCMD), where the terminology 'coding' refers to both the channel coding and space-time coding. Firstly, in order to maximize the MIMO achievable rate, an optimum spatial component interleaver is proposed. Secondly, based on the AMImaximization criterion, the optimal rotation angles of real-valued signal and complex-valued QAM signal are investigated for MIMO schemes, which are different from the SISO scheme. Thirdly, for the JCMD-ID scheme, a simple genetic algorithm (GA) to search binary convolution code (BCC) is put forward to match the rotated QAM modulation. Simulation results show that the optimized BCC-coded JCMD-ID MIMO scheme outperforms the turbo-coded BICM-ID MIMO scheme in [24] by $1.4 \mathrm{~dB}$ 
SNR gain, while the new scheme has much lower complexity.

Throughout this paper, we use bold letters to represent vectors or matrices. $(\cdot)^{T}$ and $(\cdot)^{H}$ represent transposition and conjugate transposition, respectively. $\mathrm{SNR}=E_{s} / N_{0}$, where $E_{s}$ denotes the average symbol energy per receive antenna and $N_{0}=2 \sigma^{2}$ denotes the variance of the complex Gaussian noise.

The paper is organized as follows. An improved JCMD MIMO scheme is proposed in Section 2. Theoretical analysis about the achievable rate of JCMD-MIMO for rotated real-valued signals is given in Section 3. Based on the AMI analysis, the optimal rotation angles for JCMD and JCMDID MIMO systems are presented in Section 4. Section 5 introduces an outer convolutional code search method for the optimization of JCMD-ID system with optimal rotation angle. Simulation results are presented in Section 6 on fast fading channels. Concluding remarks are offered in Section 7.

\section{System model}

Based on the BICM-TLST scheme, a system model of $N_{L}$-layer MIMO-JCMD-ID is shown in Figure 1. Perfect channel state information (CSI) is assumed to be known at both the transmitter and the receiver. In Figure 1, the iterative feedback processing is depicted in dashed lines. Without loss of generality, a rank- $L N_{R} \times N_{T}$ MIMO system with $L$ nonzero eigenvalues is assumed, where $N_{R}$ and $N_{T}$ are the number of receive and transmit antennas, respectively, and $N_{L} \leq L \leq \min \left\{N_{R}, N_{T}\right\}$. In the transmitter, $K$ information bits $\mathbf{B}=\left(b_{1}, b_{2}, \ldots, b_{K}\right)^{T}$ are encoded and interleaved to yield the coded bit sequence $\mathbf{C}=\left(c_{1}, c_{2}, \ldots, c_{N}\right)^{T}$. Afterwards, $m$-tuple coded bits are mapped to a complex symbol $x_{k}=x_{k}(I)+\mathrm{j} \cdot x_{k}(Q)$, which is chosen from a $2^{m}$-ary rotated QAM constellation set $\chi=\left\{\hat{x}_{1}, \hat{x}_{2}, \ldots, \hat{x}_{2^{m}}\right\}$ according to some optimal angle. Each symbol $x_{k}$ has one Q-component $x_{k}(Q)$ and one Icomponent $x_{k}(I)$. The rotated mapped symbol sequence is first mapped onto $N_{L}$ layers in a round robin manner. Afterwards, the conventional cyclic-shift spatial interleaver of TLST is used for different symbols on $N_{L}$ layers to exploit both the space and time diversity as the following, because the cyclic-shift spatial interleaver allows the codewords to be distributed on all layers:

$$
w_{k}^{l}=x_{k}^{i}, \quad l=(i+k-2) \bmod N_{L}+1,
$$

where $w_{k}^{l}$ denotes the $k$ th $\left(k \in \mathbf{N}^{+}\right)$symbol at the $l$ th $(l \in$ $\left[1, N_{L}\right]$ ) layer after the spatial interleaver, and $x_{k}^{i}$ denotes the $k$ th symbol at the $i$ th $\left(i \in\left[1, N_{L}\right]\right)$ layer before the interleaver. Then, a spatial Q-component interleaver is applied for the $\mathrm{Q}$ components of $N_{L}$ symbols at the same instant as the following:

$$
z_{k}^{n}(Q)=w_{k}^{l}(Q), \quad n=N_{L}-l+1,
$$

where $z_{k}^{n}$ denotes the $k$ th symbol at the $n$th $\left(n \in\left[1, N_{L}\right]\right)$ layer after the spatial $Q$ interleaver. Thus, I components keep the same layer order as before, and just Q components change the layer order. The spatial Q-component interleaver is used to make the fading of I component and that of the Q component as uncorrelated as possible in the space domain. Thus, the modulation diversity of the proposed scheme is further extended to the spatial dimension. Actually, the Q-component spatial interleaver can be different from the reverse interleaver in Equation 2 . For example, it can be a cyclic-shift interleaver as the following,

$$
z_{k}^{n}(Q)=w_{k}^{l}(Q), \quad n=\left(l \bmod N_{L}\right)+1
$$

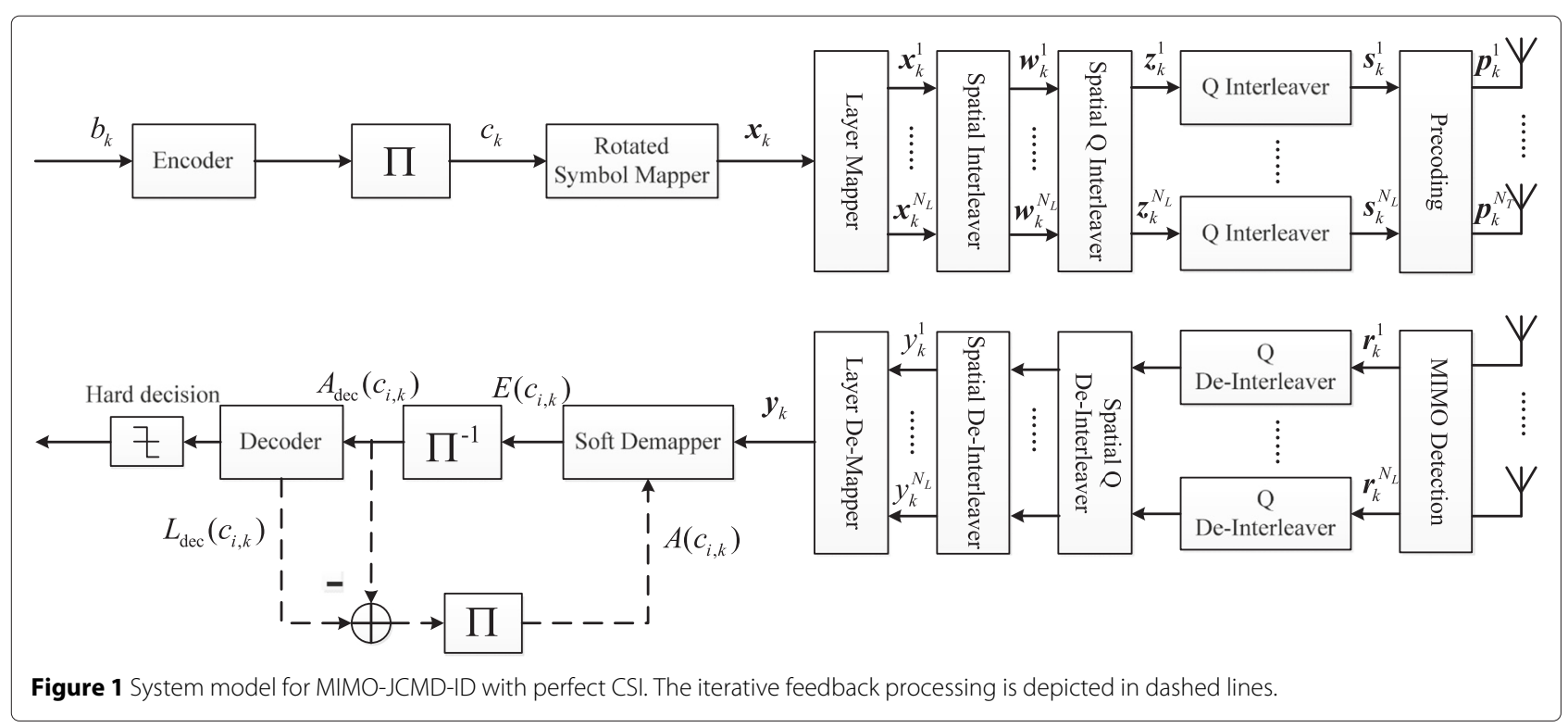


If perfect CSI is known, we prove that the reverse interleaver is better than other interleavers through the later theoretical analysis and computer simulations. If CSI is unknown at the transmitter, the cyclic-shift interleaver in Equation 3 can be used.

In order to make the fading of I component and that of the $\mathrm{Q}$ component as uncorrelated as possible in the time domain, after the spatial $\mathrm{Q}$ interleaving, $\mathrm{Q}$ components of the mapped symbols in each layer are interleaved through a time-domain pseudo S-random interleaver to reconstruct a new symbol vector $\mathbf{s}_{k}=\left[s_{k}^{1} \cdots s_{k}^{N_{L}}\right]^{T}$, where $s_{k}^{l}$ denotes the $k$ th symbol at the $l$ th layer after the component interleaving. Afterwards, the symbols are mapped onto $N_{T}$ transmit antennas via the spatial precoding and then transmitted.

The ideal precoding matrix can be obtained by singular value decomposition (SVD), which can divide MIMO channel into parallel independent SISO channels. According to the SVD criterion, the $N_{R} \times N_{T}$ MIMO channel matrix $\mathbf{H}_{k}$ can be decomposed as

$$
\mathbf{H}_{k}=\mathbf{U}_{k} \mathbf{D}_{k} \mathbf{V}_{k}{ }^{H},
$$

where the $N_{R} \times N_{R}$ matrix $\mathbf{U}_{k}$ and the $N_{T} \times N_{T}$ matrix $\mathbf{V}_{k}$ are unitary matrices. $\mathbf{D}_{k}$ is a $N_{R} \times N_{T}$ non-negative diagonal matrix with $N_{L}$ nonzero descending-order singular values, $\sqrt{\rho_{1}} \geq \sqrt{\rho_{2}} \geq \ldots \geq \sqrt{\rho_{N_{L}}}>0$, where $\rho_{i}$ is the $i$ th largest eigenvalue of $\mathbf{H}_{k} \cdot \mathbf{H}_{k}{ }^{H}$. Thus, the SVD-based linear precoding is performed as the following:

$$
\mathbf{p}_{k}=\mathbf{V}_{k} \cdot \mathbf{s}_{k} \text {. }
$$

In the receiver, the corresponding detection matrix is $\mathbf{U}_{k}^{H}$. The precoding and detection process can be expressed as linear transformations as shown in Equation 6.

$$
\begin{aligned}
\mathbf{r}_{k} & =\mathbf{U}_{k}^{H} \cdot\left(\mathbf{H}_{k} \cdot \mathbf{p}_{k}+\mathbf{n}_{k}\right) \\
& =\mathbf{U}_{k}^{H} \cdot \mathbf{H}_{k} \cdot \mathbf{V}_{k} \cdot \mathbf{s}_{k}+\mathbf{U}_{k}^{H} \cdot \mathbf{n}_{k} \\
& =\mathbf{D}_{k} \cdot \mathbf{s}_{k}+\mathbf{n}^{\prime}{ }_{k},
\end{aligned}
$$

where $\mathbf{r}_{k}=\left[r_{k}^{1} \cdots r_{k}^{N_{R}}\right]^{T}$ denotes the received symbol vector, $\mathbf{n}_{k}$ and $\mathbf{n}^{\prime}{ }_{k}$ are column vectors of $N_{R}$ complex Gaussian random variables with mean zero and variance $\sigma^{2}=\frac{N_{0}}{2}$. Thus, due to SVD, the MIMO channel can be viewed as $N_{L}$ parallel fading channels, and for $l$ th layer, the $k$ th received symbol that corresponds to $s_{k}^{l}$ in the transmitter can be expressed as

$$
r_{k}^{l}=\sqrt{\rho_{l}} \cdot s_{k}^{l}+{n^{\prime}}_{k}^{l} \text {. }
$$

After the corresponding Q-component de-interleaving in time domain for each layer and spatial Q-component de-interleaving, the $k$ th received symbol on $l$ th layer is reconstructed as $y_{k}^{l}$ that corresponds to $x_{k}^{l}$ in the transmitter. For $y_{k}^{l}$, the fading coefficients of I-component
$\lambda_{k}(I)$ and Q-component $\lambda_{k}(Q)$ are different, which can be expressed as

$$
\begin{aligned}
y_{k}^{l}(I) & =\lambda_{k}^{l}(I) x_{k}^{l}(I)+n_{k}^{l}(I) \\
y_{k}^{l}(Q) & =\lambda_{k}^{l}(Q) x_{k}^{l}(Q)+n_{k}^{l}(Q) .
\end{aligned}
$$

Assuming that the $\mathrm{Q}$ interleaver is long enough, each coordinate of the symbol after the Q de-interleaving can be regarded as suffering from independent fading coefficients. The equivalent coded modulation (CM) channel for JCMD system before sending to the soft demapper can be modeled as

$$
Y_{k}^{l}(\eta)=\Lambda_{k}^{l}(\eta) X_{k}^{l}(\eta)+N_{k}^{l}(\eta), \eta \in\{I, Q\}, l \in\left[1, N_{L}\right],
$$

where $N_{k}^{l}(I)$ and $N_{k}^{l}(Q)$ are identically independently distributed (i.i.d.) Gaussian noise random variables with zero mean and variance of $\sigma^{2}=\frac{N_{0}}{2}$. For MIMO fading channels, $\Lambda_{k}^{l}(I)$ and $\Lambda_{k}^{l}(Q)$ are singular values of corresponding sub-channels. This is in net contrast with respect to SISO scheme where the fading coefficients are Rayleigh distributed. That means the modulation diversity of proposed scheme is further extended to the spatial dimension. By denoting $\mathbf{X}=\left[\mathbf{X}_{1}, \ldots, \mathbf{X}_{N_{L}}\right]^{T}$, $\mathbf{Y}=\left[\mathbf{Y}_{1}, \ldots, \mathbf{Y}_{N_{L}}\right]^{T}, \mathbf{N}=\left[\mathbf{N}_{1}, \ldots, \mathbf{N}_{N_{L}}\right]^{T}$, and $\Lambda=$ $\operatorname{diag}\left(\Lambda_{1}, \ldots, \Lambda_{N_{L}}\right)$ representing a $\left(2 N_{L} \times 2 N_{L}\right)$ diagonal matrix, the channel model in Equation 9 can be written in the matrix form as $\mathbf{Y}=\Lambda \mathbf{X}+\mathbf{N}$, where $\mathbf{X}_{l}=\left[X_{k}^{l}(I), X_{k}^{l}(Q)\right], \mathbf{Y}_{l}=\left[Y_{k}^{l}(I), Y_{k}^{l}(Q)\right], \mathbf{N}_{l}=$ $\left[N_{k}^{l}(I), N_{k}^{l}(Q)\right]$, and $\Lambda_{l}=\operatorname{diag}\left(\Lambda_{k}^{l}(I), \Lambda_{k}^{l}(Q)\right)$.

After that, symbols on multiple layers are reassembled as symbol streams $\mathbf{y}=\left[y_{1}, y_{2} \cdots\right]^{T}$ through the layer demapping. A serial concatenation of a soft-in-softout rotated symbol demapper and a channel decoder are employed to approach the maximum likelihood (ML) receiver performance. For JCMD-ID, the iterative demapping and decoding scheme is an application of the turbo decoder principle. The soft demapper calculates the extrinsic value $E\left(c_{i, k}\right)$ of bit $c_{i, k}$ which corresponds to the $i$ th $(i=1,2, \ldots, m)$ bit of the received symbol $y_{k}$ as follows:

$$
E\left(c_{i, k}\right)=L\left(c_{i, k}\right)-A\left(c_{i, k}\right),
$$

where $L\left(c_{i, k}\right) \triangleq \ln \frac{P\left(c_{i, k}=0 \mid y_{k}\right)}{P\left(c_{i, k}=1 \mid y_{k}\right)}$ is the log-likelihood ratio (LLR) and $A\left(c_{i, k}\right) \triangleq \ln \frac{P\left(c_{i, k}=0\right)}{P\left(c_{i, k}=1\right)}$ is a priori L-value. Based on Bayes' theorem, we can write 


$$
E\left(c_{i, k}\right)=\ln \frac{\sum_{\substack{\hat{x} \in x_{i} \\ \hat{c}_{i}=0}} P\left(y_{k} \mid x_{k}=\hat{x}\right) \exp \left\{\sum_{\substack{j=1, j \neq i}}^{m}\left[(-1)^{\hat{c}_{j}} \frac{A\left(c_{j, k}\right)}{2}\right]\right\}}{\sum_{\substack{\hat{x} \in x_{,} \\ \hat{c}=1}} P\left(y_{k} \mid x_{k}=\hat{x}\right) \exp \left\{\sum_{\substack{j=1, j \neq i}}^{m}\left[(-1)^{\hat{c}_{j}} \frac{A\left(c_{j, k}\right)}{2}\right]\right\}},
$$

where $\hat{c}_{i}$ is the $i$ th bit corresponding to symbol $\hat{x}$. For the fading channel, the conditional probability is given by

$$
P\left(y_{k} \mid x_{k}=\hat{x}\right)=\frac{1}{2 \pi \sigma^{2}} \exp \left(-\frac{\left(y_{k}^{I}-\lambda_{k}^{I} \hat{x}^{I}\right)^{2}+\left(y_{k}^{Q}-\lambda_{k}^{Q} \hat{x}^{Q}\right)^{2}}{2 \sigma^{2}}\right) .
$$

To the complexity, by applying Max-Log-MAP algorithm, Equation 11 can be simplified as

$$
\begin{gathered}
E\left(c_{i, k}\right)=\max _{\substack{\hat{x} \in \times, \hat{c}_{i, k}=0}}\left\{\Omega_{k}(\hat{x})-\sum_{\substack{j=1, j \neq i}}^{m}\left[(-1)^{\hat{c}_{j, k}} \frac{A\left(c_{j, k}\right)}{2}\right]\right\}- \\
\max _{\substack{\hat{x} \in x_{0} \\
\hat{c}_{i, k}=1}}\left\{\Omega_{k}(\hat{x})-\sum_{\substack{j=1, j \neq i}}^{m}\left[(-1)^{\hat{c}_{j, k}} \frac{A\left(c_{j, k}\right)}{2}\right]\right\},
\end{gathered}
$$

where $\Omega_{k}(\hat{x})=-\frac{\left(y_{k}^{I}-\lambda_{k}^{I} \hat{x}^{I}\right)^{2}+\left(y_{k}^{Q}-\lambda_{k}^{Q} \hat{x}^{Q}\right)^{2}}{2 \sigma^{2}}$

For the JCMD system without the iterative demapping and decoding, $A\left(c_{i, k}\right)=0$. Finally, the decoder can utilize the extrinsic values to decode information bits.

In the transmitter, compared with the conventional BICM, the JCMD scheme introduces extra constellation rotation and Q-component interleavers. Constellation rotation does not increase the complexity, because the rotated symbol mapping can be implemented through look-up table operations as the same as the conventional modulation without rotation. Q-component interleavers also can be implemented by the low-complexity indexbased look-up table operations.

In the receiver, the soft rotated demapping operation of JCMD system is the same as that of BICM system, which is shown in Equation 11. Q-component de-interleavers also can be implemented by the simple reverse index-based look-up table operations.

\section{Theoretical analysis of the achievable rate for rotated real-valued signals}

Firstly, since real-valued signals are elementary, we analyze the real-valued transmit signals in MIMO systems.
Lemma 1. For any constant rank-2 MIMO fading channel with real-valued transmit signals, the constant achievable rate of JCMD-MIMO is not less than that of BICMMIMO with the conventional uniform power allocation. If and only if the constant eigenvalues are identical, both of them are equal, otherwise the former is greater than the latter.

Proof. A simple rank-2 MIMO case with two eigenvalues $\rho_{1}$ and $\rho_{2}$ is illustrated in Figure 2. Due to the well-known SVD, BICM-MIMO can be viewed as two parallel fading channels with two eigenvalues $\rho_{1}$ and $\rho_{2}$ for spatial layer 1 and layer 2 , respectively. Two fading amplitude coefficients of layer 1 and layer 2 are the corresponding singular values $\sqrt{\rho_{1}}$ and $\sqrt{\rho_{2}}$, respectively, as shown in the left half of Figure 2. Thus, given the same transmit power $\frac{P}{2}$ on each layer for one real-valued symbol, the received symbol power of layer 1 and that of layer 2 are $\frac{\rho_{1} P}{2}$ and $\frac{\rho_{2} P}{2}$, respectively, where $P$ is the total transmit power for two layers. So, according to Shannon's theory, the achievable rate of BICM-MIMO is shown as the following:

$$
\begin{aligned}
C_{1} & =\frac{W}{2} \cdot \log _{2}\left[\left(1+\frac{\rho_{1} P}{2 \sigma^{2}}\right)\left(1+\frac{\rho_{2} P}{2 \sigma^{2}}\right)\right] \\
& =\frac{W}{2} \cdot \log _{2}\left(1+\frac{\rho_{1}+\rho_{2}}{2 \sigma^{2}} P+\frac{\rho_{1} \rho_{2}}{4 \sigma^{4}} P^{2}\right),
\end{aligned}
$$

where $W$ is the channel bandwidth, $\sigma^{2}$ is the variance of AWGN. As we know, in order to achieve the capacity in the Equation 14, the transmit signals should be Gaussian distributed. Note that the rotation does not change the achievable rate for the BICM-MIMO scheme without the I/Q-component interleaver.

For the JCMD-MIMO scheme, we consider the $\frac{\pi}{4}$ rotated real-valued transmit signal, which is rotated by $\frac{\pi}{4}$ compared with the conventional real-valued signal. Due to the orthogonal modulation, the transmit powers of I component and that of $\mathrm{Q}$ component on each layer are both $\frac{P}{4}$. For JCMD-MIMO, after the spatial Q-component deinterleaver at the receiver, the fading amplitude coefficient of I component is different from that of $\mathrm{Q}$ component in one symbol, that is to say, one is $\sqrt{\rho_{1}}$, and another is $\sqrt{\rho_{2}}$. Thus, the received power of I component is also different from that of $\mathrm{Q}$ component, that is to say, one is $\frac{\rho_{1} P}{4}$, and another is $\frac{\rho_{2} P}{4}$. So, the total received symbol power is $\frac{\left(\rho_{1}+\rho_{2}\right) P}{4}$ both for layer 1 and layer 2 . Thus, JCMD-MIMO can be viewed as two parallel fading channels with identical fading amplitude coefficient $\sqrt{\frac{\rho_{1}+\rho_{2}}{2}}$ for both layer 1 and layer 2, as shown in the right half of Figure 2. In the receiver, after the phase compensation, the received signal also can be viewed as the real-valued signal with the power 


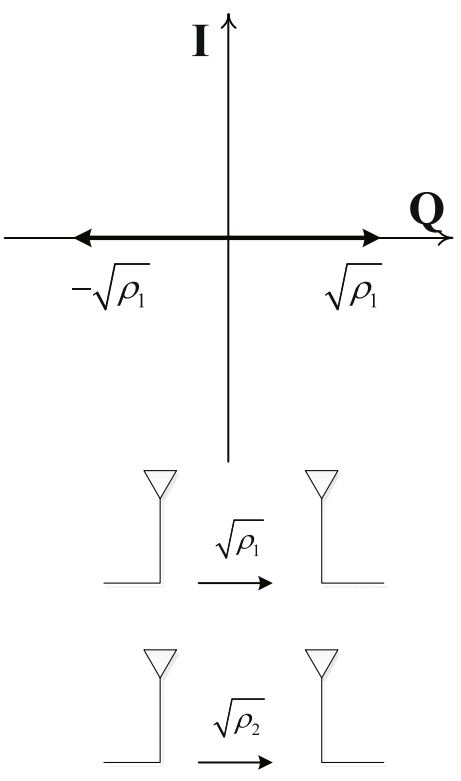

(a) BICM-MIMO
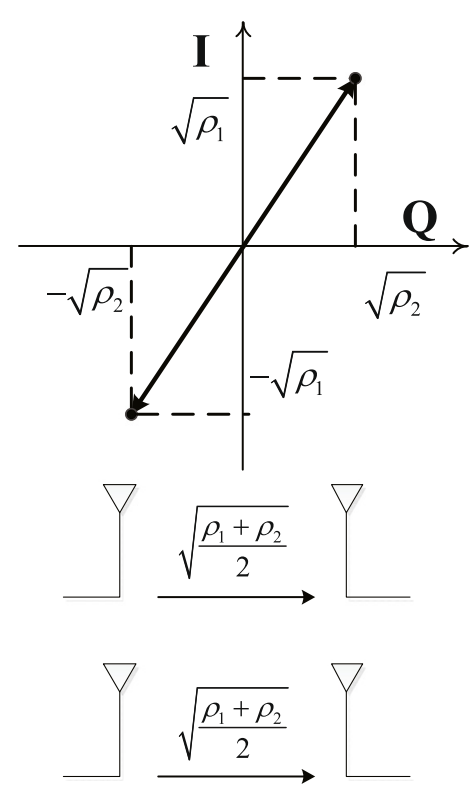

(b) JCMD-MIMO

Figure 2 Achievable rates of conventional BICM-MIMO and JCMD-MIMO system. (a) BICM-MIMO can be viewed as two parallel fading channels with two eigenvalues $\rho_{1}$ and $\rho_{2}$. (b) JCMD-MIMO can be viewed as two parallel fading channels with identical fading amplitude coefficient $\sqrt{\frac{\rho_{1}+\rho_{2}}{2}}$ for both layers.

$\frac{\left(\rho_{1}+\rho_{2}\right) P}{4}$. Therefore, the achievable rate of JCMD-MIMO is shown as the following:

$$
\begin{aligned}
C_{2} & =\frac{W}{2} \cdot \log _{2}\left[1+\frac{\left(\rho_{1}+\rho_{2}\right)}{4 \sigma^{2}} P\right]^{2} \\
& =\frac{W}{2} \cdot \log _{2}\left[1+\frac{\rho_{1}+\rho_{2}}{2 \sigma^{2}} P+\frac{\left(\rho_{1}+\rho_{2}\right)^{2}}{16 \sigma^{4}} P^{2}\right] .
\end{aligned}
$$

So, comparing Equation 14 with 15, it is easy to come to the conclusion: $C_{2} \geq C_{1}$

If and only if $\rho_{2}=\rho_{1}, C_{2}=C_{1}$.

Furthermore, we will prove that $\frac{\pi}{4}$ is the optimum rotation angle for real-valued transmit signals in the JCMDMIMO scheme. Let us consider a general $\theta$-rotated realvalued transmit signal, which is rotated by $\theta$ compared with the conventional real-valued signal. Thus, the transmit power of I component and that of $\mathrm{Q}$ component on layer 1 are $\frac{P}{2} \cos ^{2} \theta$ and $\frac{P}{2} \sin ^{2} \theta$, respectively, and the total transmit power on layer 1 is also $\frac{P}{2}$. Therefore, the received power of I component and that of Q component are $\frac{\rho_{2} P}{2} \cos ^{2} \theta$ and $\frac{\rho_{1} P}{2} \sin ^{2} \theta$, respectively. So, the total received symbol power on layer 1 is $\frac{\left(\rho_{1} \sin ^{2} \theta+\rho_{2} \cos ^{2} \theta\right)}{2} P$. Likewise, the total received symbol power on layer 2 is $\frac{\left(\rho_{2} \sin ^{2} \theta+\rho_{1} \cos ^{2} \theta\right)}{2} P$. Thus, we can get the following achievable rate:

$$
\begin{aligned}
C(\theta) & =\frac{W}{2} \cdot \log _{2}\left\{\left[1+\frac{\left(\rho_{1} \sin ^{2} \theta+\rho_{2} \cos ^{2} \theta\right)}{2 \sigma^{2}} P\right] \cdot\left[1+\frac{\left(\rho_{1} \cos ^{2} \theta+\rho_{2} \sin ^{2} \theta\right)}{2 \sigma^{2}} P\right]\right\} \\
& =\frac{W}{2} \cdot \log _{2}\left[1+\frac{\rho_{1}+\rho_{2}}{2 \sigma^{2}} P+\left(\rho_{1} \rho_{2}+\frac{\sin ^{2}(2 \theta)\left(\rho_{1}-\rho_{2}\right)^{2}}{4}\right) \frac{P^{2}}{4 \sigma^{4}}\right] .
\end{aligned}
$$

Obviously, when $\theta=0$, the achievable rate is minimum as Equation 14; and when $\theta=\frac{\pi}{4}$, the achievable rate is maximum as Equation 15.

According to Lemma 1, we can come to the following theorem:

Theorem 1. For any actual rank-2 MIMO fading channel with real-valued transmit signals, the ergodic achievable rate of JCMD-MIMO is greater than that of BICMMIMO with the conventional uniform power allocation.

Proof. For any actual rank-2 MIMO fading channel, the ergodic achievable rate is the mathematical average expectation of the above constant achievable rate over all possible channel eigenvalue realization. Generally speaking, the equal eigenvalue realization is only a small probability event. The case that the channel eigenvalues are different must be existed. According to Lemma 1 , the ergodic achievable rate of JCMD-MIMO is greater 
than that of BICM-MIMO with the conventional uniform power allocation.

Generally speaking, assuming a rank- $L$ MIMO with a descending-order eigenvalue vector $\overline{\boldsymbol{\rho}}=\left\{\rho_{1}, \rho_{2}, \ldots, \rho_{L}\right\}$, $\mathrm{Q}$-component interleaver only changes the order of $\mathrm{Q}$ components on $L$ layers to another eigenvalue vector $\bar{\zeta}=$ $\left\{\zeta_{1}, \zeta_{2}, \ldots, \zeta_{L}\right\}$, where $\bar{\zeta}$ is just another arrangement order of $\bar{\rho}$ corresponding to the output order of Q-component spatial interleaver. Hence, due to the orthogonal modulation, JCMD-MIMO can be viewed as $L$ parallel fading channels with an eigenvalue vector $\bar{v}=\frac{\bar{\rho}+\bar{\zeta}}{2}$. So, the achievable rate of rank- $L$ JCMD-MIMO is shown as the following:

$$
\begin{aligned}
C_{L}(\bar{\zeta}) & =\frac{W}{2} \cdot \sum_{i=1}^{L} \log _{2}\left[1+\frac{\left(\rho_{i}+\xi_{i}\right)}{2 \sigma^{2} L} P\right] \\
& =\frac{W}{2} \cdot \log _{2}\left[\prod_{i=1}^{L}\left(1+\frac{\left(\rho_{i}+\xi_{i}\right)}{2 \sigma^{2} L} P\right)\right],
\end{aligned}
$$

where $P$ is the total transmit power for $L$ layers. Hence, the optimum problem of the achievable rate is to find the optimum $\bar{\zeta}$ so as to maximize $C_{L}$. We can reach the following theorem:

Theorem 2. To maximize the achievable rate of rank- $L$ JCMD-MIMO with a descending-order eigenvalue vector $\bar{\rho}=\left\{\rho_{1}, \rho_{2}, \ldots, \rho_{L}\right\}$, the optimum Q-component interleaver vector $\overline{\boldsymbol{\omega}}=\left\{\rho_{L}, \rho_{L-1}, \ldots, \rho_{1}\right\}$, that is to say, $\overline{\boldsymbol{\omega}}$ should be in ascending order, which is just in reverse order of $\bar{\rho}$.

Proof. Assuming someone claims to find a non-increasing-order vector $\bar{\rho}^{\prime}$ other than $\overline{\boldsymbol{\omega}}$ to have maximum achievable rate $C_{L}\left(\bar{\rho}^{\prime}\right)$, we must can find a pair of $\left\{\rho_{i}^{\prime}, \rho_{i+1}^{\prime}\right\}$ from $\bar{\rho}^{\prime}$ to satisfy $\rho_{i}^{\prime}>\rho_{i+1}^{\prime}$. And then, we can construct a new vector $\eta=\left\{\rho_{1}^{\prime}, \rho_{2}^{\prime}, \ldots, \rho_{i-1}^{\prime}, \rho_{i+1}^{\prime}, \rho_{i}^{\prime} \rho_{i+2}^{\prime}, \ldots, \rho_{L}^{\prime}\right\}$, which only changes the order of $\left\{\rho_{i}^{\prime}, \rho_{i+1}^{\prime}\right\}$ from $\bar{\rho}^{\prime}$. So, we can compute the difference of $\frac{C_{L}(\eta)-C_{L}\left(\overline{\boldsymbol{\rho}}^{\prime}\right)}{W / 2}$,

$$
\begin{aligned}
\frac{C_{L}(\boldsymbol{\eta})-C_{L}\left(\overline{\boldsymbol{\rho}}^{\prime}\right)}{W / 2}= & \sum_{k=1}^{L} \log _{2}\left[1+\frac{\left(\rho_{k}+\eta_{k}\right)}{2 \sigma^{2} L} P\right]-\sum_{k=1}^{L} \log _{2}\left[1+\frac{\left(\rho_{k}+\rho_{k}^{\prime}\right)}{2 \sigma^{2} L} P\right] \\
= & \log _{2}\left[1+\frac{\left(\rho_{i}+\eta_{i}\right)}{2 \sigma^{2} L} P\right]+\log _{2}\left[1+\frac{\left(\rho_{i+1}+\eta_{i+1}\right)}{2 \sigma^{2} L} P\right] \\
& -\log _{2}\left[1+\frac{\left(\rho_{i}+\rho_{i}^{\prime}\right)}{2 \sigma^{2} L} P\right]-\log _{2}\left[1+\frac{\left(\rho_{i+1}+\rho_{i+1}^{\prime}\right)}{2 \sigma^{2} L} P\right] \\
= & \log _{2}\left[1+\frac{\left(\rho_{i}+\rho_{i+1}^{\prime}\right)}{2 \sigma^{2} L} P\right]+\log _{2}\left[1+\frac{\left(\rho_{i+1}+\rho_{i}^{\prime}\right)}{2 \sigma^{2} L} P\right] \\
& -\log _{2}\left[1+\frac{\left(\rho_{i}+\rho_{i}^{\prime}\right)}{2 \sigma^{2} L} P\right]-\log _{2}\left[1+\frac{\left(\rho_{i+1}+\rho_{i+1}^{\prime}\right)}{2 \sigma^{2} L} P\right] .
\end{aligned}
$$

Let a constant $c=\frac{P}{2 \sigma^{2} L}$, and then,

$$
\frac{C_{L}(\boldsymbol{\eta})-C_{L}\left(\overline{\boldsymbol{\rho}}^{\prime}\right)}{W / 2}=\log _{2} \frac{M_{1}}{M_{2}},
$$

where

$$
\begin{aligned}
M_{1}= & +c\left(\rho_{i}+\rho_{i}^{\prime}+\rho_{i+1}+\rho_{i+1}^{\prime}\right) \\
& +c^{2}\left(\rho_{i}+\rho_{i+1}^{\prime}\right)\left(\rho_{i+1}+\rho_{i}^{\prime}\right)>0, \\
M_{2}= & +c\left(\rho_{i}+\rho_{i}^{\prime}+\rho_{i+1}+\rho_{i+1}^{\prime}\right) \\
& +c^{2}\left(\rho_{i}+\rho_{i}^{\prime}\right)\left(\rho_{i+1}+\rho_{i+1}^{\prime}\right)>0 .
\end{aligned}
$$

So, the difference

$$
M_{1}-M_{2}=c^{2}\left(\rho_{i}-\rho_{i+1}\right)\left(\rho_{i}^{\prime}-\rho_{i+1}^{\prime}\right) .
$$

Because $\rho_{i}>\rho_{i+1}$ and $\rho_{i}^{\prime}>\rho_{i+1}^{\prime}$.

So, $M_{1}-M_{2}>0$; Thus, $\frac{M_{1}}{M_{2}}>1$; and then, $C_{L}(\eta)-$ $C_{L}\left(\overline{\boldsymbol{\rho}}^{\prime}\right)>0$.

That is to say, we find a counter-example $\boldsymbol{\eta}$ to have more achievable rate than $C_{L}\left(\bar{\rho}^{\prime}\right)$, which contradicts the assumption of maximum achievable rate $C_{L}\left(\bar{\rho}^{\prime}\right)$. Therefore, $\bar{\omega}$ should be in ascending order, which is just in reverse order of $\bar{\rho}$.

So, in Section 2, the reverse Q-component spatial interleaver is applied as Equation 2 so as to have the maximum achievable rate. In fact, if $L$ is an even number, the rank$L$ JCMD-MIMO with the reverse Q-component spatial interleaver can be viewed as $\frac{L}{2}$ parallel pairs of rank-2 JCMD-MIMO with eigenvalues $\left\{\rho_{i}, \rho_{L+1-i}\right\}$, where $i \in$ $\left[1, \frac{L}{2}\right]$. Likewise, if $L$ is an odd number, it can be viewed as the parallel combination of one SISO fading channel with eigenvalue $\left\{\rho_{\frac{L+1}{2}}\right\}$ and $\frac{L-1}{2}$ pairs of rank-2 JCMDMIMO with eigenvalues $\left\{\rho_{i}, \rho_{L+1-i}\right\}$, where $i \in\left[1, \frac{L-1}{2}\right]$. Thus, the largest eigenvalue layer couples with the smallest eigenvalue layer, the second largest eigenvalue layer couples with the second smallest eigenvalue layer, and so on. Actually, BICM-MIMO is also one special case of JCMD-MIMO when $\bar{\zeta}=\bar{\rho}$.

Theorem 3. As for the achievable rate of a rank- $L$ JCMD-MIMO with a descending-order eigenvalue vector $\bar{\rho}=\left\{\rho_{1}, \rho_{2}, \ldots, \rho_{L}\right\}$, the upper bound is $C_{L}(\overline{\boldsymbol{\omega}})$, where $\bar{\omega}$ is in reverse order of $\bar{\rho}$, and the lower bound is the BICM-MIMO achievable rate $C_{L}(\bar{\rho})$.

Proof. According to Theorem 2, the maximum achievable rate is $C_{L}(\overline{\boldsymbol{\omega}})$, we can get $C \leq C_{L}(\overline{\boldsymbol{\omega}})$. In addition, $C_{L}(\overline{\boldsymbol{\rho}})$ is the minimum achievable rate, which can be proved by the similar math skill as follows.

Assuming someone claims to find a non-descendingorder vector $\bar{\rho}^{\prime}$ other than $\bar{\rho}$ to have minimum achievable rate $C_{L}\left(\overline{\boldsymbol{\rho}}^{\prime}\right)$, we must can find a pair of $\left\{\rho_{i}^{\prime}, \rho_{i+1}^{\prime}\right\}$ from $\overline{\boldsymbol{\rho}}^{\prime}$ to satisfy $\rho_{i}^{\prime}<\rho_{i+1}^{\prime}$. And then, we can construct a new 
vector $\eta=\left\{\rho_{1}^{\prime}, \rho_{2}^{\prime}, \ldots, \rho_{i-1}^{\prime}, \rho_{i+1}^{\prime}, \rho_{i}^{\prime}, \rho_{i+2}^{\prime}, \ldots, \rho_{L}^{\prime}\right\}$, which only changes the order of $\left\{\rho_{i}^{\prime}, \rho_{i+1}^{\prime}\right\}$ from $\bar{\rho}^{\prime}$. So, we can compute the difference of $\frac{C_{L}(\eta)-C_{L}\left(\bar{\rho}^{\prime}\right)}{W / 2}$,

$$
\begin{aligned}
\frac{C_{L}(\boldsymbol{\eta})-C_{L}\left(\overline{\boldsymbol{\rho}}^{\prime}\right)}{W / 2}= & \sum_{k=1}^{L} \log _{2}\left[1+\frac{\left(\rho_{k}+\eta_{k}\right)}{2 \sigma^{2} L} P\right]-\sum_{k=1}^{L} \log _{2}\left[1+\frac{\left(\rho_{k}+\rho_{k}^{\prime}\right)}{2 \sigma^{2} L} P\right] \\
= & \log _{2}\left[1+\frac{\left(\rho_{i}+\eta_{i}\right)}{2 \sigma^{2} L} P\right]+\log _{2}\left[1+\frac{\left(\rho_{i+1}+\eta_{i+1}\right)}{2 \sigma^{2} L} P\right] \\
& -\log _{2}\left[1+\frac{\left(\rho_{i}+\rho_{i}^{\prime}\right)}{2 \sigma^{2} L} P\right]-\log _{2}\left[1+\frac{\left(\rho_{i+1}+\rho_{i+1}^{\prime}\right)}{2 \sigma^{2} L} P\right] \\
= & \log _{2}\left[1+\frac{\left(\rho_{i}+\rho_{i+1}^{\prime}\right)}{2 \sigma^{2} L} P\right]+\log _{2}\left[1+\frac{\left(\rho_{i+1}+\rho_{i}^{\prime}\right)}{2 \sigma^{2} L} P\right] \\
& -\log _{2}\left[1+\frac{\left(\rho_{i}+\rho_{i}^{\prime}\right)}{2 \sigma^{2} L} P\right]-\log _{2}\left[1+\frac{\left(\rho_{i+1}+\rho_{i+1}^{\prime}\right)}{2 \sigma^{2} L} P\right] .
\end{aligned}
$$

Let a constant $c=\frac{P}{2 \sigma^{2} L}$, and then,

$$
\frac{C_{L}(\boldsymbol{\eta})-C_{L}\left(\overline{\boldsymbol{\rho}}^{\prime}\right)}{W / 2}=\log _{2} \frac{M_{1}}{M_{2}},
$$

where

$$
\begin{aligned}
M_{1}= & 1+c\left(\rho_{i}+\rho_{i}^{\prime}+\rho_{i+1}+\rho_{i+1}^{\prime}\right) \\
& +c^{2}\left(\rho_{i}+\rho_{i+1}^{\prime}\right)\left(\rho_{i+1}+\rho_{i}^{\prime}\right)>0, \\
M_{2}= & 1+c\left(\rho_{i}+\rho_{i}^{\prime}+\rho_{i+1}+\rho_{i+1}^{\prime}\right) \\
& +c^{2}\left(\rho_{i}+\rho_{i}^{\prime}\right)\left(\rho_{i+1}+\rho_{i+1}^{\prime}\right)>0 .
\end{aligned}
$$

So, the difference

$$
M_{1}-M_{2}=c^{2}\left(\rho_{i}-\rho_{i+1}\right)\left(\rho_{i}^{\prime}-\rho_{i+1}^{\prime}\right) .
$$

Because $\rho_{i}>\rho_{i+1}$ and $\rho_{i}^{\prime}<\rho_{i+1}^{\prime}$.

So, $M_{1}-M_{2}<0$; Thus, $\frac{M_{1}}{M_{2}}<1$; and then, $C_{L}(\eta)-$ $C_{L}\left(\overline{\boldsymbol{\rho}}^{\prime}\right)<0$.

That is to say, we find a counter-example $\eta$ to have small achievable rate than $C_{L}\left(\bar{\rho}^{\prime}\right)$, which contradicts the assumption of minimum achievable rate $C_{L}\left(\bar{\rho}^{\prime}\right)$. Therefore, $C_{L}(\overline{\boldsymbol{\rho}})$ is the minimum achievable rate.

\section{AMI analysis for complex-valued QAM signals}

For practical communication systems, the transmit signal $\mathbf{X}$ usually belongs to a finite alphabet (constellation signal set), such as the complex-valued QAM signal. AMI also varies with the rotation angle of QAM signals in the MIMO system. Assuming equiprobable QAM constellation inputs and the independent Rayleigh fading channel, the AMI of the coded modulation (CM) MIMO system before the demapper is called CM-AMI, which is irrelevant to the labeling and defined as [2]

$$
\begin{aligned}
I_{\mathrm{CM}} & =I(\mathbf{X} ; \mathbf{Y} \mid \Lambda) \\
& =\sum_{l=1}^{N_{L}}\left\{m-E_{\mathbf{x}_{l}, \mathbf{y}_{l}, \lambda_{l}}\left[\log _{2} \frac{\sum_{\hat{\mathbf{x}} \in \chi} P\left(\mathbf{y}_{l} \mid \hat{\mathbf{x}}, \lambda_{l}\right)}{P\left(\mathbf{y}_{l} \mid \mathbf{x}_{l}, \lambda_{l}\right)}\right]\right\} .
\end{aligned}
$$

The AMI after the demapper is called BICM-AMI, which is relevant to the labeling and defined as [2]

$$
\begin{aligned}
I_{\mathrm{BICM}} & =\sum_{k=1}^{m} I\left(C_{k} ; \mathbf{Y} \mid \mathbf{\Lambda}\right) \\
& =\sum_{l=1}^{N_{L}}\left\{m-\sum_{k=1}^{m} E_{c_{k}, \mathbf{y}_{l}, \lambda_{l}}\left[\log _{2} \frac{\sum_{\hat{\mathbf{x}} \in \chi} p\left(\mathbf{y}_{l} \mid \hat{\mathbf{x}}, \lambda_{l}\right)}{\sum_{\hat{\mathbf{x}} \in \chi, \hat{c}_{k}=c_{k}} p\left(\mathbf{y}_{l} \mid \hat{\mathbf{x}}, \lambda_{l}\right)}\right]\right\} .
\end{aligned}
$$

AMI analysis is an effective method to calculate its achievable rate. From Equation 28 , because $I(\mathbf{X} ; \mathbf{Y} \mid \Lambda)$ is independent from labeling, it implies that the AMI of CM system is not related to the labeling. However, for the BICM system, the BICM-AMI strongly depends on the labeling. Extensive literature has proven that Gray labeling is optimal for the non-ID BICM system in the AWGN channel [25]. Monte Carlo simulation techniques are useful tools to get the expectation of a complex function by ergodicity of the random variables [26]. The expectation operations in Equations 28 and 29 can be evaluated by using the Monte Carlo simulation techniques. Based on Equation 9, we can get the received symbol before the soft demapper by generating the random coded bits $c_{k}$ (corresponding to the modulated symbol $x_{k}^{l}$ on each layer), the random fading coefficient $\lambda_{k}^{l}$ (SVD of i.i.d. Rayleigh-distributed random channel matrix $\mathbf{H}_{k}$ ), and the i.i.d. Gaussian noise random variable $n_{k}^{l}$. Thus, by using the Monte Carlo simulation techniques, we can estimate the expectation values of Equations 28 and 29 by ergodicity of $\left(c_{k}, \mathbf{H}_{k}, n_{k}^{l}\right)$, where $P\left(\mathbf{y}_{l} \mid \mathbf{x}_{l}, \lambda_{l}\right)$ can be calculated as Equation 12. As for complex-valued QAM signals, the optimum rotation angle usually is different from the value of the real-valued signals, and we can get it by maximizing AMI.

Figure 3 shows how $2 \times 2$ MIMO CM-AMI varies with the rotation angle $\theta$ for real-valued binary phase shift keying (BPSK) symbols. Since $I(\mathbf{X} ; \mathbf{Y} \mid \mathbf{\Lambda})=I\left(C_{k} ; \mathbf{Y} \mid \mathbf{\Lambda}\right)$, the $I_{\mathrm{CM}}$ and $I_{\mathrm{BICM}}$ are the same for BPSK. Both for low and high SNR, e.g., at SNR $=-5 \mathrm{~dB}$ and $\mathrm{SNR}=-15 \mathrm{~dB}$, the optimal rotation angles are always $\theta=45^{\circ}$, which coincides with our analysis in Section 3. 


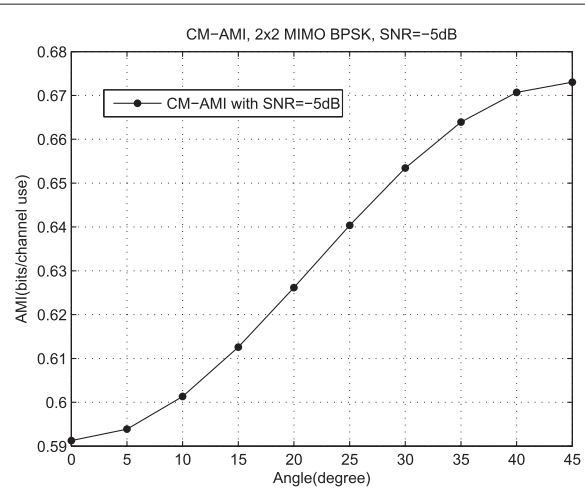

(a) $S N R=-5 d B$

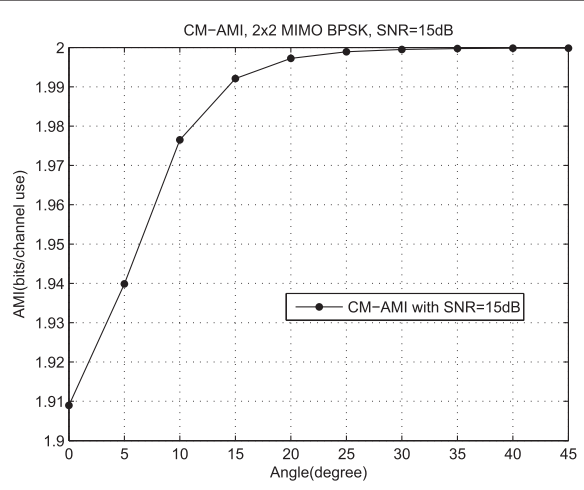

(b) $S N R=15 d B$

Figure $32 \times 2$ MIMO CM-AMI ICM vs. rotation angle $\theta$ for BPSK over Rayleigh fading channels. (a) $S N R=-5 \mathrm{~dB}$. (b) $S N R=15 \mathrm{~dB}$.

Figures 4 and 5 show how $4 \times 4$ MIMO CM-AMI and BICM-AMI vary with the rotation angle $\theta$ for QPSK and 16QAM, respectively. The AMIs of proposed systems with two kinds of spatial Q-component interleavers (reverse and cyclic-shift) are plotted in the same figures. Examples of low SNR and high SNR are presented. In Figures 4 and 5, for both spatial Q interleaving algorithms, the optimal rotation angles just have slight difference in high SNR. For CM-AMI, the optimal angle of QPSK at $\mathrm{SNR}=-3 \mathrm{~dB}$ is $45^{\circ}$, while the optimal angle at $\mathrm{SNR}=$ $11 \mathrm{~dB}$ is about $30^{\circ}$ and $29^{\circ}$ for the cyclic-shift interleaver and the reverse interleaver, respectively. For BICM-AMI, the optimal angle of QPSK at SNR $=-3 \mathrm{~dB}$ is $0^{\circ}$, while the optimal angle at $\mathrm{SNR}=11 \mathrm{~dB}$ is about $26^{\circ}$ and $27^{\circ}$ for the cyclic-shift interleaver and the reverse interleaver, respectively.

Based on the AMI maximization criterion, the optimal angle corresponds to the maximum AMI value. Figure 6 shows the CM-AMI and BICM-AMI curves that correspond to the optimal angles in Figures 4 and 5 for $4 \times 4$
MIMO systems. In Figure 6, CM-AMI and BICM-AMI for the reverse interleaver described in Equation 2 are always not less than that of the cyclic-shift interleaver described in Equation 3 both for QPSK and 16QAM. These observations concide well with Theorem 2.

Based on maximizing AMI, we can also obtain the relationship between optimal angle and SNR. The optimal angles for CM and BICM systems vary with SNR for QPSK and 16QAM, which is plotted in Figure 7. The optimal rotation angle of CM is always bigger than that of BICM. When SNR increases, the optimal angle of CM becomes smaller, but the optimal angle of BICM becomes bigger.

For a given modulation order $m$, according to the AMI value $I$ corresponding to optimal rotation angle in Figure 6, the optimal code rate for a given SNR can be calculated as $R=\frac{I}{m \cdot N_{L}}$. Therefore, we can get the relationship between the optimal code rate $R$ and SNR, which is shown in Figure 8.

Thus, for a given operating SNR, we can obtain the optimum rotation angle in Figure 7 and the optimal

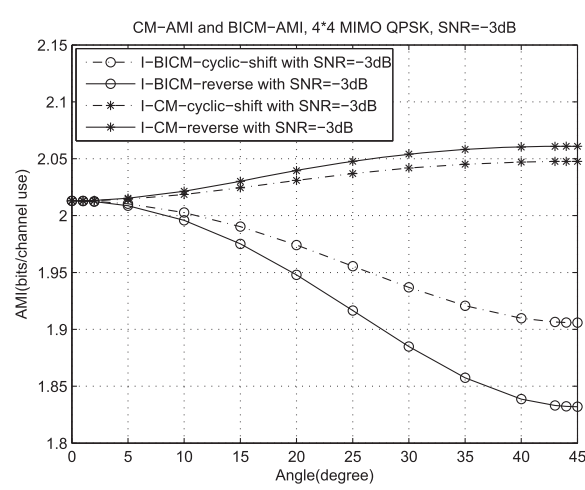

(a) $\mathrm{SNR}=-3 \mathrm{~dB}$

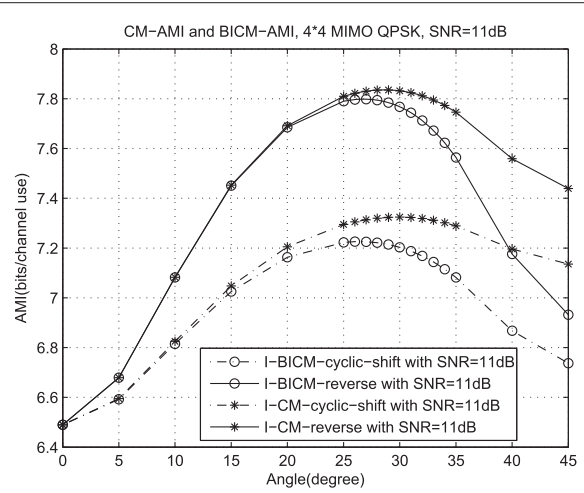

(b) $S N R=11 \mathrm{~dB}$

Figure $44 \times 4$ MIMO CM-AMI ICM and BICM-AMI IBICM vs. rotation angle $\theta$ for QPSK over Rayleigh fading channels. (a) $S N R=-3 \mathrm{~dB}$. (b) $\mathrm{SNR}=11 \mathrm{~dB}$. 


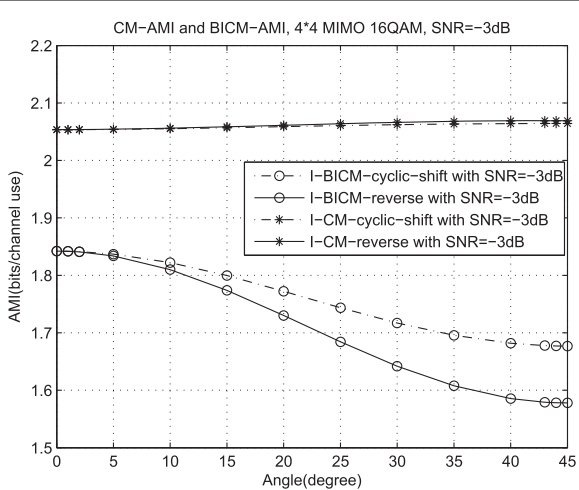

(a) $S N R=-3 d B$

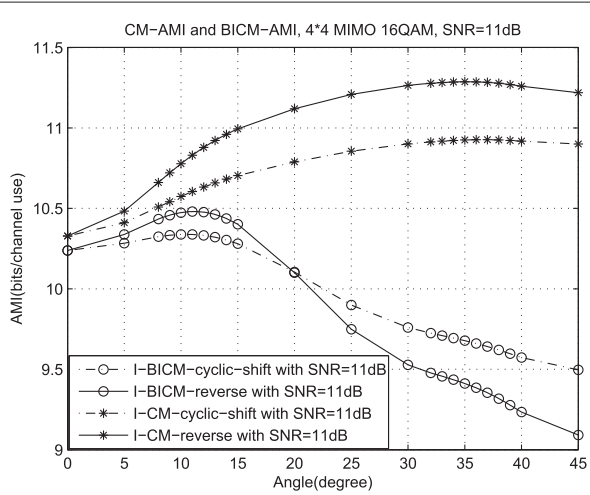

(b) $S N R=11 d B$

Figure $54 \times 4$ MIMO CM-AMI I IM and BICM-AMI IBICM Vs. rotation angle $\theta$ for 16QAM over Rayleigh fading channels. (a) $S N R=-3 \mathrm{~dB}$. (b) $\mathrm{SNR}=11 \mathrm{~dB}$.

code rate in Figure 8. Furthermore, we can get the relationship between code rate $R$ and optimal rotation angle, which is shown in Figure 9 for QPSK and 16QAM. It provides a good reference to select an optimal rotation angle for a given code rate. For instance, for code rate $=0.5$, the optimal angles for $4 \times 4$ MIMOBICM QPSK and 16QAM are $18^{\circ}$ and $0^{\circ}$, respectively. According to Figures 7 and 9, at low SNR or low code rate, non-rotation is the best for BICM, which implies that the channel coding dominates the BICM performance, while $45^{\circ}$ rotation is the best for CM, which indicates that the rotation symmetry affects the CM performance. However, at high SNR or high code rate, the signal space diversity dominates the performance both for
BICM and CM, so BICM-AMI can approach CM-AMI, and the optimum angle of BICM with the optimum reverse spatial interleaver is also close to that of $\mathrm{CM}$ with the same interleaver. Note that the rotation angle is just related to the code rate and modulation. So, in practice, the rotation symbol mapper can be implemented through a look-up table that is calculated and stored in advance for a given modulation order and code rate, which is the same as the conventional symbol mapper. Hence, it does not introduce additional processing complexity and delay.

\section{Code design for JCMD-ID system}

For the non-ID BICM system, Gray mapping has been proved to be optimal. Based on the optimal rotation angle

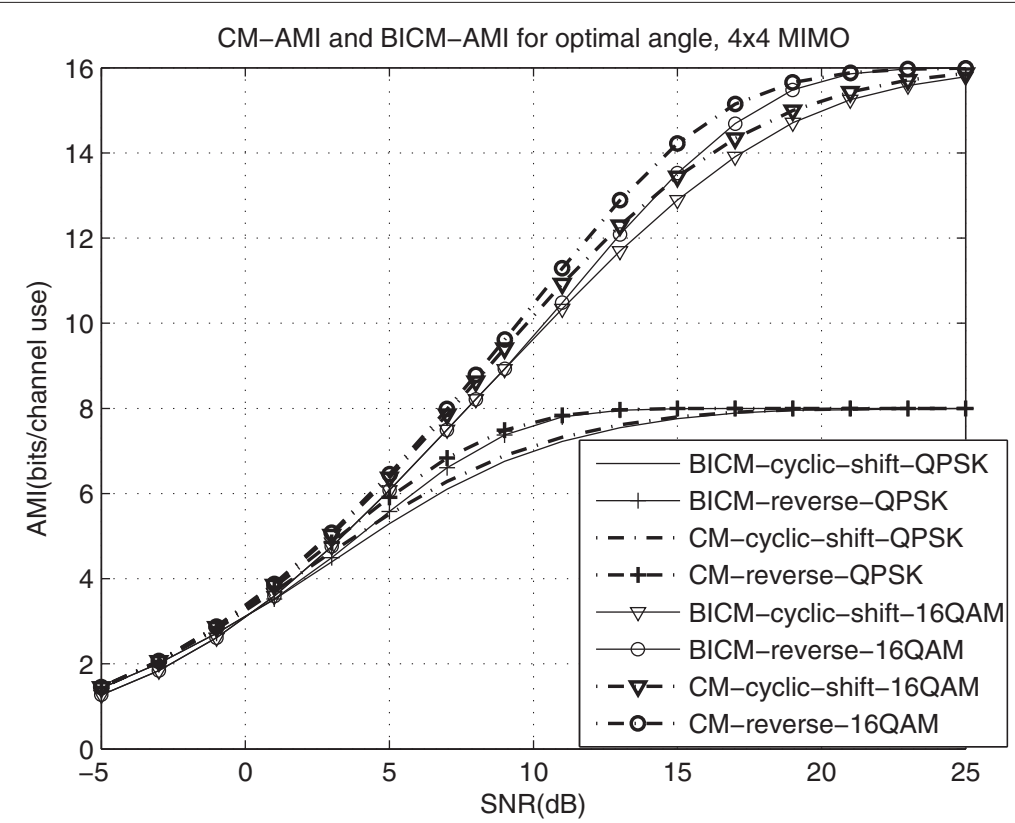

Figure $6 \mathrm{CM}-\mathrm{AMI}$ and $\mathrm{BICM}-\mathrm{AMI}$ corresponding to the optimal angles for $4 \times 4 \mathrm{MIMO}$. 


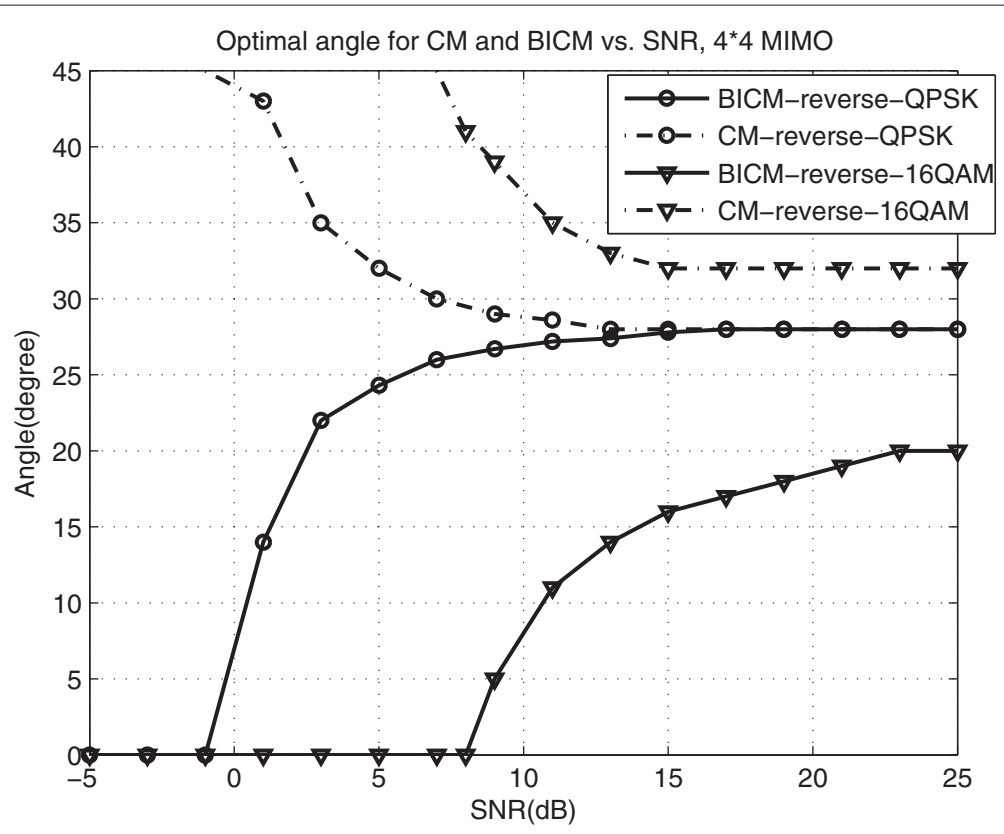

Figure 7 Optimal angle $\theta$ vs. SNR for QPSK and 16QAM over $4 \times 4$ Rayleigh fading channels.

obtained by maximizing BICM-AMI and Gray mapping, the non-ID JCMD system only needs to consider the optimization of channel codes to achieve excellent performance. For the JCMD-ID system, besides the optimal rotation angle, it is also very crucial to choose a pair of a well-matched labeling and an outer channel code by some joint optimization.
The convergence behavior of the iterative demodulation and decoding can be analyzed by the EXIT chart method, which can describe the flow of extrinsic information between the demodulator and the decoder [27-29]. Several advantages of EXIT charts are summarized in [29]. The inputs to the demapper are the noise-corrupted channel observations and the a priori knowledge $A\left(c_{k}\right)$ on

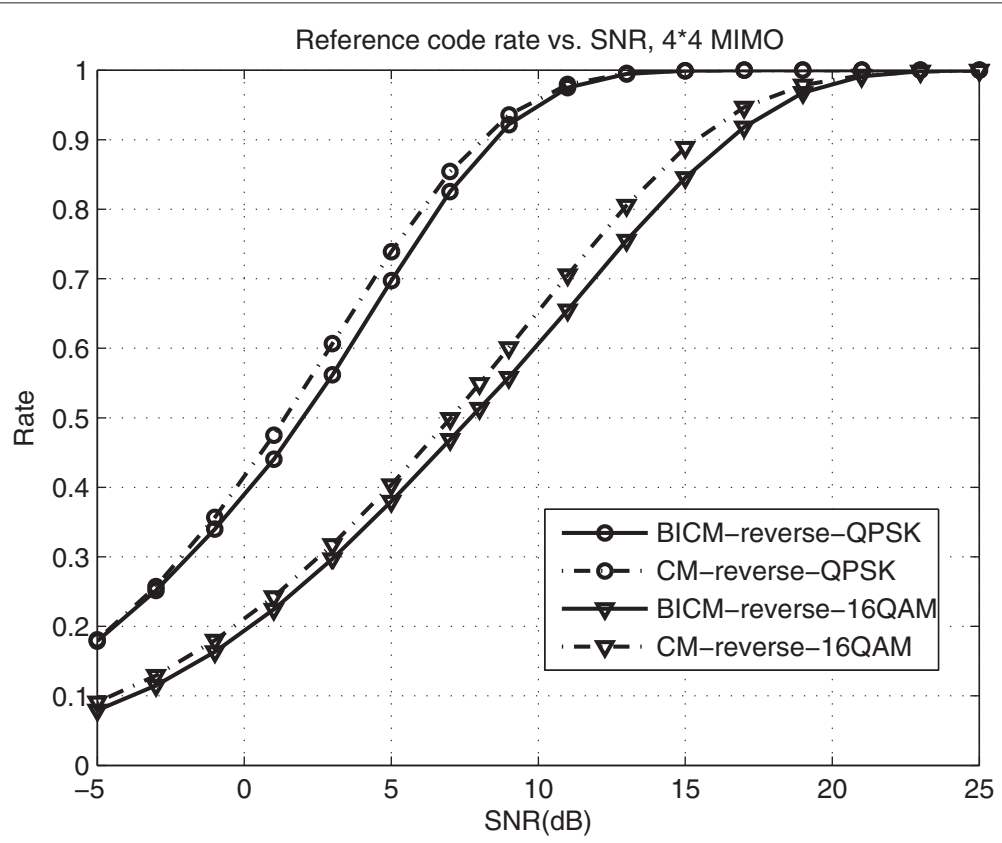

Figure 8 Code rate vs. SNR for QPSK and 16QAM over $4 \times 4$ Rayleigh fading channels. 


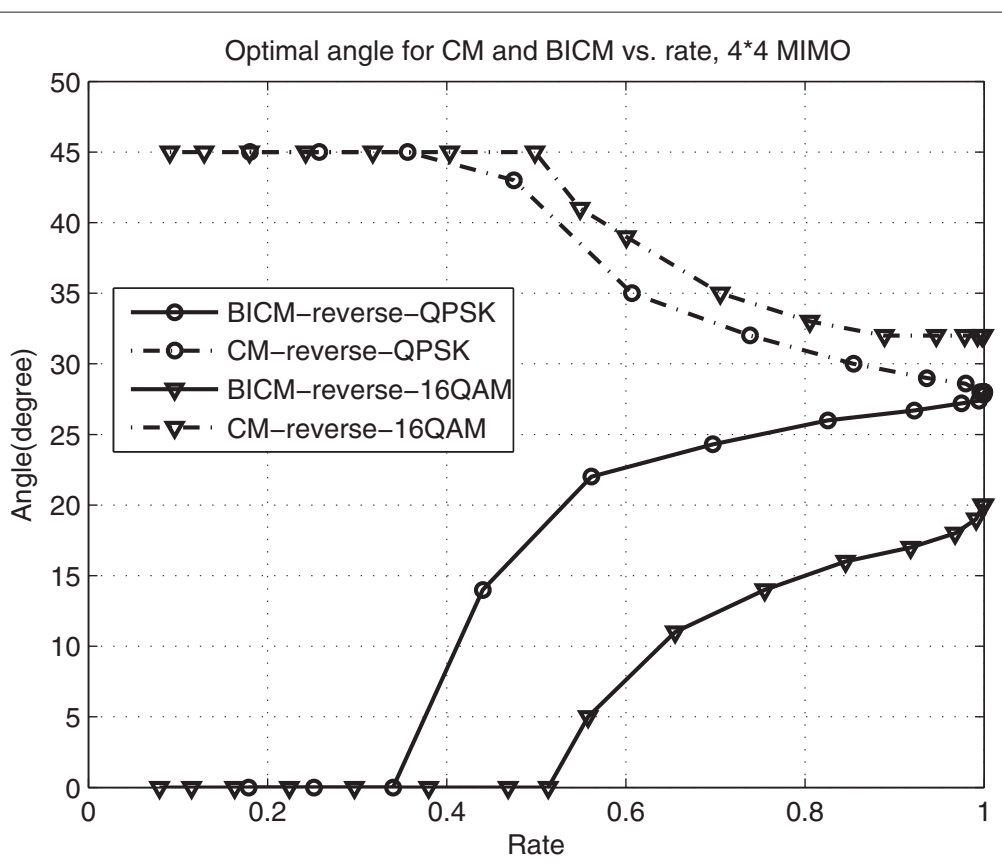

Figure 9 Optimal angle $\theta$ vs. code rate $R$ for QPSK and 16QAM over $4 \times 4$ Rayleigh fading channels.

the unmapped bits. The demapper outputs channel and extrinsic information $E\left(c_{k}\right)$. According to Equations 12 and 19 in [27], the mutual information $I_{A 1}=I\left(c_{k} ; A\left(c_{k}\right)\right)$ $\left(0 \leq I_{A 1} \leq 1\right)$ between transmitted unmapped bits and the $L$-values $A\left(c_{k}\right)$ can be written as

$$
\begin{aligned}
I_{A 1}= & \frac{1}{2} \sum_{x=-1,1} \int_{-\infty}^{\infty} p_{A\left(c_{k}\right)}\left(\xi \mid c_{k}=x\right) \cdot \log _{2} \\
& \times \frac{2 p_{A\left(c_{k}\right)}\left(\xi \mid c_{k}=x\right)}{p_{A\left(c_{k}\right)}\left(\xi \mid c_{k}=-1\right)+p_{A\left(c_{k}\right)}\left(\xi \mid c_{k}=1\right)} d \xi .
\end{aligned}
$$

The mutual information $I_{E 1}=I\left(c_{k} ; E\left(c_{k}\right)\right)\left(0 \leq I_{E 1} \leq 1\right)$ can be written as

$$
\begin{aligned}
I_{E 1}= & \frac{1}{2} \sum_{x=-1,1} \int_{-\infty}^{\infty} p_{E\left(c_{k}\right)}\left(\xi \mid c_{k}=x\right) \cdot \log _{2} \\
& \times \frac{2 p_{E\left(c_{k}\right)}\left(\xi \mid c_{k}=x\right)}{p_{E\left(c_{k}\right)}\left(\xi \mid c_{k}=-1\right)+p_{E\left(c_{k}\right)}\left(\xi \mid c_{k}=1\right)} d \xi .
\end{aligned}
$$

In [27], it turns out that the a priori input $A\left(c_{k}\right)$ is almost Gaussian distributed. Additionally, large interleavers keep the a priori $L$-values $A\left(c_{k}\right)$ fairly uncorrelated over many iterations. Hence, it seems appropriate to model the $a$ priori input $A\left(c_{k}\right)$ by applying an independent Gaussian random variable with variance $\sigma_{A 1}^{2}$ and mean zero (see Equations 1 to 10 in [27]). Thus, the conditional probability density function can be written as

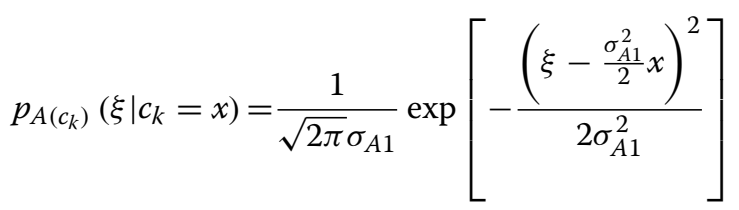

The mutual information $I_{E 1}=I\left(c_{k} ; E\left(c_{k}\right)\right)$ can be viewed as a function of $I_{A 1}$, SNR, and the rotation angle $\theta$, i.e,

$$
I_{E_{1}}=T_{1}\left(I_{A_{1}}, S N R, \theta\right) \text {. }
$$

The function $T_{1}$ can not be expressed in a closed form. For a given value of the input mutual information $I_{A 1}=$ $I\left(c_{k} ; A\left(c_{k}\right)\right)\left(0 \leq I_{A 1} \leq 1\right)$ to the demodulator, to compute $T_{1}\left(I_{A_{1}}, S N R, \theta\right)$, the distributions $p_{E\left(c_{k}\right)}\left(\xi \mid c_{k}=x\right)$ are most conveniently determined by the Monte Carlo simulation (histogram measurements) proposed in [27,28]. The convenient method has been verified to allow an accurate prediction of the SNR-decoding threshold with low complexity [27].

On the other hand, the extrinsic transfer characteristics of the decoder can describe the input/output relationship between the input $I_{A 2}$ and the output $I_{E 2}$, which is independent of SNR value. It can be computed by assuming the a priori input to be Gaussian distributed and applying the same method as used for the demapper characteristic $T_{1}$. Therefore, the transfer characteristic of the decoder is denoted by

$$
I_{E_{2}}=T_{2}\left(I_{A_{2}}\right) .
$$


Qiuliang et al. [16] verified that the SSD technique can affect the demapper's EXIT curve for the SISO BICM-ID SSD system. For the MIMO system, we analyze the effect of the SSD technique to different demapper labelings. Different 16QAM labelings (natural, Gray, and reference [16]) are shown in Figure 10. For the 1/2-rate coded 16QAM $4 \times 4$ MIMO system, the corresponding 16QAM EXIT curves with $45^{\circ}$ rotation and non-rotation are depicted in Figure 11. The doping technique is used for errorfloor removal [30] and the doping rate $P=100$. For Natural and Gray labelings, the demappers' EXIT curves with rotation have a larger slope than that without rotation. For the referenced labeling, the demapper's EXIT curve with rotation is always above the curve without rotation.

Demapper-matched code design is very crucial for the JCMD-ID MIMO system with rotation. In order to approach the capacity, based on EXIT chart, we propose an optimization method of outer channel codes to match with a given demapper for the JCMD-ID system. We choose the BCC as the channel code. For a given SNR, if two EXIT curves of the demapper and the decoder do not intersect, the iterative decoding can converge, otherwise it cannot converge. Thus, the SNR which makes the two EXIT curves tangent is the SNR convergence threshold, which is also called pinch-off SNR. The objective of JCMD-ID optimization is to find the outer channel code that has the lowest SNR convergence threshold to match with the demapper.

We define the optimization function as follows.

$$
\alpha\left(\mathbf{G}\left(N_{\mathrm{Reg}}\right), S N R\right)=\min _{I_{n} \in[0,1], n=1,2, \ldots, \mathrm{Num}}\left[T_{1}\left(I_{n}\right)-T_{2}^{-1}\left(I_{n}\right)\right],
$$

$$
\overline{\mathrm{SNR}}=\underset{\mathrm{SNR}}{\arg \min }\left[\alpha\left(\mathbf{G}\left(N_{\mathrm{Reg}}\right), \mathrm{SNR}\right)>0\right],
$$

where $\mathbf{G}\left(N_{\mathrm{Reg}}\right)$ denotes the generator polynomial of BCC with $N_{\text {Reg }}$ registers. Num is the number of selected statistical samples. $\overline{\text { SNR }}$ is the pinch-off SNR. For $\frac{1}{N_{\text {out }}}$ rate
BCC, the objective is to find the optimum $\mathbf{G}_{\text {Opt. }}\left(N_{\text {Reg }}\right)$ with the lowest pinch-off SNR from $\left(2^{N_{\text {Reg }}+1}-1\right)^{N_{\text {out }}}$ generator polynomial candidates. However, the exhaustive searching of a channel code to well match the demapper's EXIT curve is trivial, especially for $N_{\text {Reg }}$ is large.

GA is an efficient optimization algorithm, which is stochastic search techniques based on the mechanism of natural selection and natural genetics [31]. In GA, a genetic representation is required for the individuals in a population. Generator polynomials of BCC $\mathbf{G}\left(N_{\mathrm{Reg}}\right)=$ $\left[\mathbf{g}_{1}, \mathbf{g}_{2}, \ldots, \mathbf{g}_{N_{\text {out }}}\right]_{2}$ inherently provides a $\left(N_{\text {Reg }}+1\right) \times N_{\text {out }}-$ length binary string $\mathbf{S}_{g}=<\mathbf{g}_{1}, \mathbf{g}_{2}, \ldots, \mathbf{g}_{N_{\text {out }}}>$. $\mathbf{g}_{i}$ is the $\left(N_{\text {Reg }}+1\right)$-length binary generator polynomial corresponding to $i$ th $\left(1 \leq i \leq N_{\text {out }}\right)$ output. Based on the genetic algorithm, an optimization method is proposed as follows.

Step 1: Initial population. Set the current iterative number (number of generations) $N_{\text {pop }}=0$, the number of candidate generator polynomials (population size) $N_{g}$, the maximum iterative number $N_{\text {max }}$, crossover probability $P_{c}$, and mutation probability $P_{m} . N_{g}$ binary strings $\mathbf{S}_{g}^{i}=<\mathbf{g}_{1}^{i}, \mathbf{g}_{2}^{i}, \ldots, \mathbf{g}_{N_{\text {out }}}^{i}>$ that correspond to the candidate polynomials are randomly initialized, which are denoted by a set $\mathbf{C}^{N_{\text {pop }} \text {, where }}$ $\mathbf{g}_{1}^{i}, \mathbf{g}_{2}^{i}, \ldots, \mathbf{g}_{N_{\text {out }}}^{i} \in\left[1,2^{N_{\text {Reg }}+1}\right), 1 \leq i \leq N_{g}$. Step 2: Selection. Reduce the SNR by small steps $\Delta_{\mathrm{SNR}}$ (e.g., $0.1 \mathrm{~dB}$ ) and compute the pinch-off SNRs $\overline{\mathrm{SNR}_{i}}$ of all the candidate generator polynomials $\mathbf{G}_{i}\left(N_{\mathrm{Reg}}\right)=\left[\mathbf{g}_{1}^{i}, \mathbf{g}_{2}^{i}, \ldots, \mathbf{g}_{N_{\text {out }}}^{i}\right]_{2}$ in population, where $<\mathbf{g}_{1}^{i}, \mathbf{g}_{2}^{i}, \ldots, \mathbf{g}_{N_{\text {out }}}^{i}>\in \mathbf{C}^{N_{\text {pop }}, 1} 1 \leq i \leq N_{g}$. We use the pinch-off SNR to measure the fitness. The fitness function is associated with the maximum pinch-off $\mathrm{SNR} \overline{\mathrm{SNR}}_{\max }=\max _{1 \leq i \leq N_{g}}\left\{\overline{\mathrm{SNR}}_{i}\right\}$, shown as

$$
f\left(\mathbf{S}_{g}^{i}\right)=\overline{\mathrm{SNR}}_{\max }(\mathrm{dB})+0.1-\overline{\mathrm{SNR}}_{i}(\mathrm{~dB})
$$

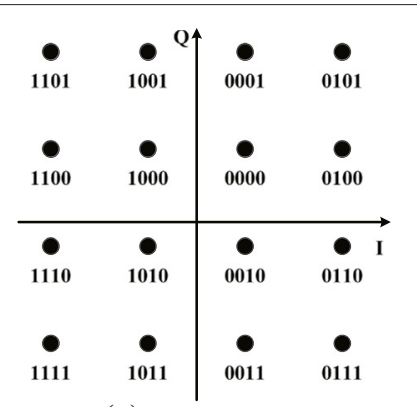

(a) Gray labeling

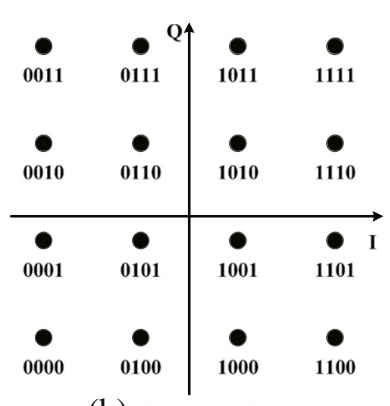

(b) Natural labeling

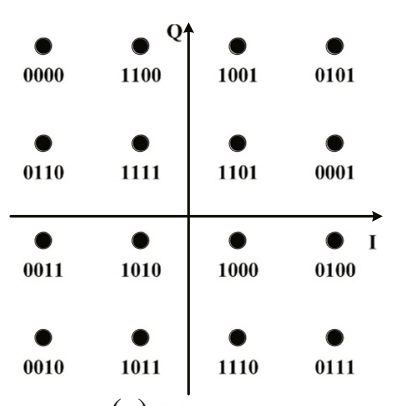

(c) Reference

Figure 10 16QAM labelings. (a) Gray labeling. (b) Natural labeling. (c) The reference labeling in [16]. 


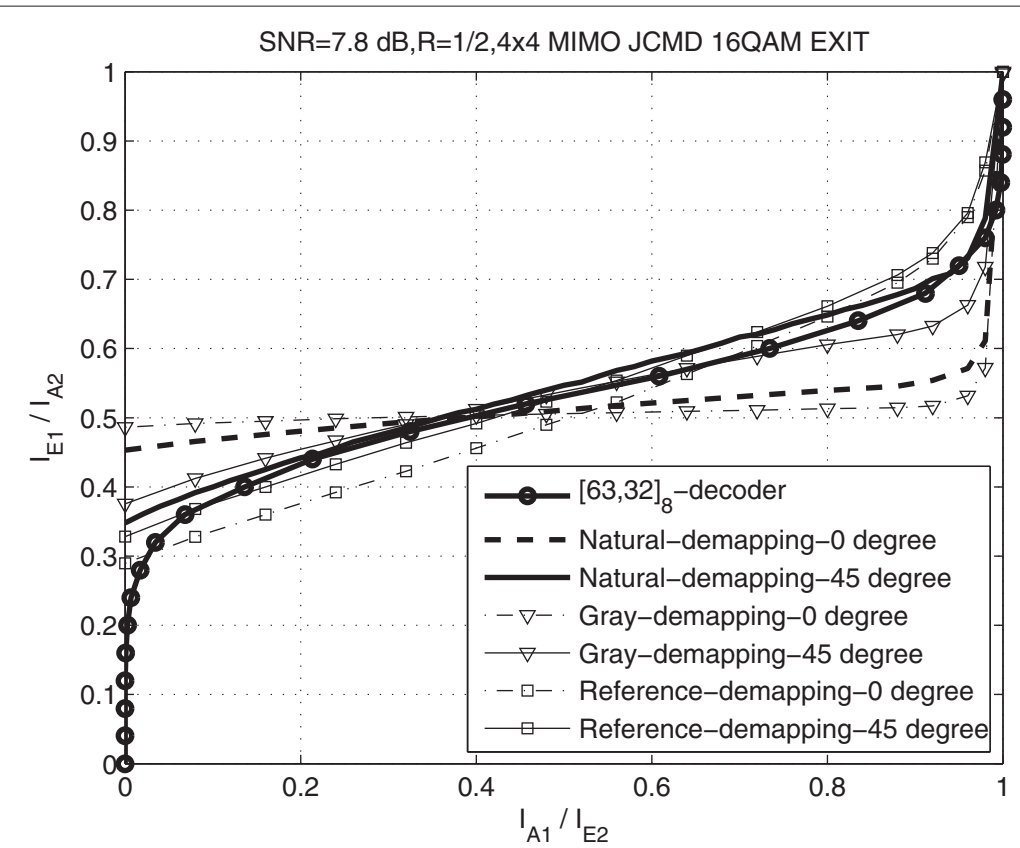

Figure 11 EXIT chart analysis for 16QAM at SNR $=7.8 \mathrm{~dB}$.

$N_{g}$ individuals are selected to breed a new generation with probability proportional to the fitness value.

The probability that $\mathbf{S}_{g}^{i}$ is selected is $P(i)=\frac{f\left(\mathbf{S}_{g}^{i}\right)}{\sum_{k=1}^{N_{g}} f\left(\mathbf{S}_{g}^{k}\right)}$.

Based on roulette wheel selection (RWS) algorithm, the $t$ th $\left(1 \leq t \leq N_{g}\right)$ individual selection follows the steps below.

$A$. Generate a uniform random number $\chi(t)$, $\chi(t) \in[0,1]$.

B. If $\sum_{i=0}^{k-1} P(i) \leq \chi(t)<\sum_{i=1}^{k} P(i)\left(1 \leq k \leq N_{g}\right)$, $P(0)=0, \mathbf{S}_{g}^{k}$ is selected.

Step 3: Crossover. For two adjacent selected individuals, a random number $\kappa_{c}$ from the range $[0,1]$ is generated. Only when $\kappa_{c}<P_{c}$, the crossover operator is carried out. The crossover point is selected randomly. All bits beyond that point in either string are swapped between the two parent individuals, and then two children individuals are obtained.

Step 4: Mutation. For each individuals, a random number $\kappa_{m}$ from the range $[0,1]$ is generated. Only when $\kappa_{m}<P_{m}$, the mutation operator is carried out through one bit flip at random mutation position.

Step 5: Judgment. $N_{\text {pop }}=N_{\text {pop }}+1$. After step $2 \sim 4$, a new population $\mathbf{C}^{N_{\text {pop }}+1}$ is formed. If

$N_{\text {pop }}<N_{\text {max }}$, then go to step 2 , otherwise stop. The generator polynomial with the lowest pinch-off SNR in $\mathbf{C}^{N_{\text {pop }}+1}$ is chosen as the optimum one.
Using the method above, a rate-half $\mathrm{BCC}$ code with $N_{\text {Reg }}=5$ is optimized for the natural labeling 16QAM JCMD-ID system. Based on the AMI analysis in Section 4, the optimal rotation angle for 0.5 rate 16QAM JCMDID system is $45^{\circ}$. The optimal generator polynomial is $[63,32]_{8}$. As shown in Figure 11, the natural labeling demapper's EXIT curve with $45^{\circ}$ rotation keeps a narrow open tunnel with that of $\mathrm{BCC}$ decoder, while its non-rotation curve intersects with the decoder, which shows the effect of rotation to the ID system. The other two labeling demappers' EXIT curves with and without rotation always intersects with the decoder, so the Gray and reference labelings are not suitable for the BCC decoder. Therefore, the natural labeling with $45^{\circ}$ rotation matches well with the $[63,32]_{8}$ BCC code and has the best performance.

\section{Simulation result}

\subsection{Results of non-ID JCMD system on fast fading channels}

For the non-ID JCMD MIMO system, the optimal Gray labeling and powerful DVB-T2 LDPC coding are used to achieve excellent performance [32]. The optimal rotation angle is obtained by maximizing BICM-AMI in Figure 9. The size of coded block $N$ is 64,800 bits. For the LDPC decoder, the log-belief propagation (BP) algorithm with 30 maximum iterations is utilized. In order to ensure the fairness of the comparison, the SVD precoding is implemented for both the conventional BICM MIMO system and proposed JCMD MIMO system. 


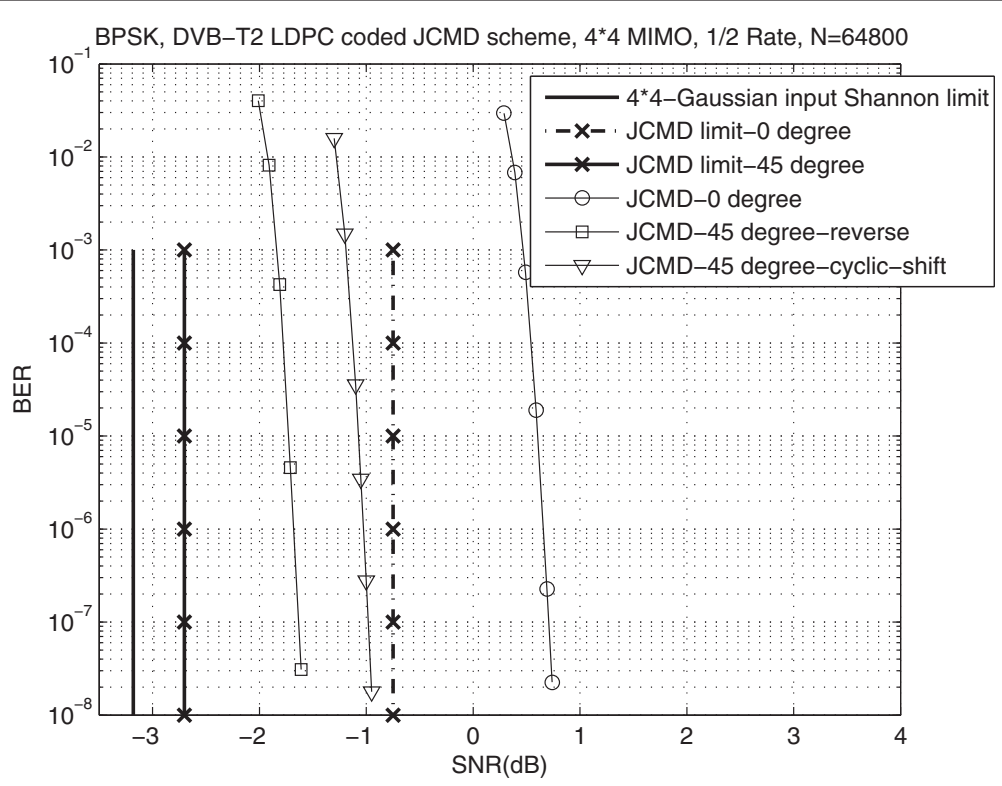

Figure 12 BER performance of rate-1/2 DVB-T2 LDPC coded, BPSK $4 \times 4$ JCMD MIMO systems on fast fading channels.

Figure 12 shows the bit error rate (BER) performance comparisons of rate-1/2 LDPC-coded BPSK MIMO systems on the independent fast Rayleigh fading channel. The channel fading coefficients on each symbol are independent identical distributed Rayleigh random variables with the variance 1 . BPSK is also a simple real-valued signal, so the optimal rotation angle is chosen as $45^{\circ}$. For $4 \times 4$ MIMO systems, JCMD with $45^{\circ}$ rotation obtains significant SNR gains as compared with JCMD without rotation. For the target $\mathrm{BER}=10^{-5}$, it can achieve $2.2 \mathrm{~dB}$ SNR gain. Note that the spatial interleaver, spatial Q-component interleaver, and time Q-component interleaver are all

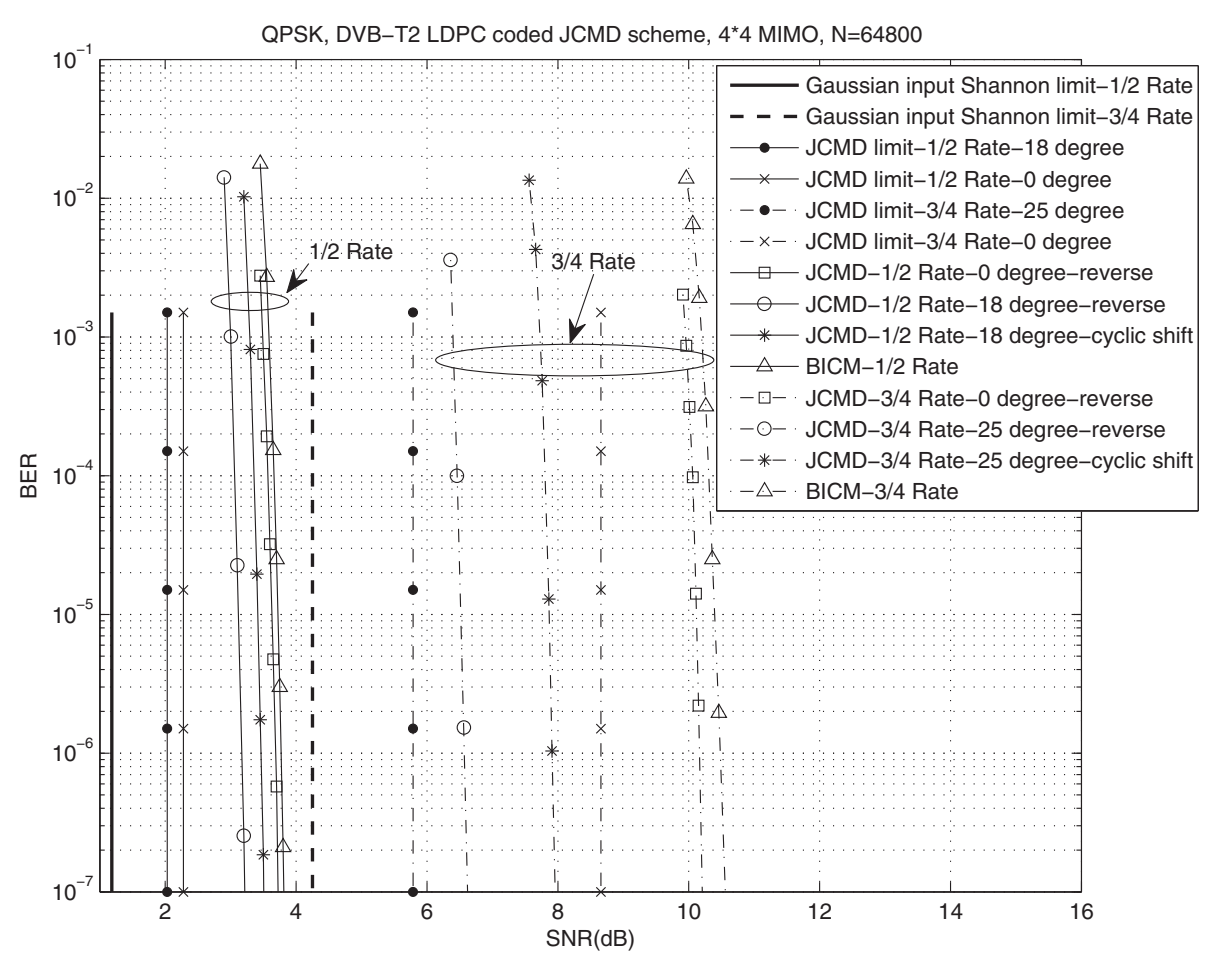

Figure 13 BER performance of rate-1/2 and 3/4 DVB-T2 LDPC coded, QPSK $4 \times 4$ JCMD MIMO systems on fast fading channels. 
Table 1 SNR gains and gaps to the capacity for JCMD $4 \times 4$ MIMO systems

\begin{tabular}{lll}
\hline Parameters & $\begin{array}{l}\text { Gains compared with } \\
\text { BICM system (dB) }\end{array}$ & $\begin{array}{l}\text { Gaps to the JCMD/Gaussian } \\
\text { input Shannon limit (dB) }\end{array}$ \\
\hline BPSK, 1/2-rate & 2.2 & $1.0 / 1.5$ \\
QPSK, 1/2-rate & 0.6 & $1.1 / 1.9$ \\
QPSK, 3/4-rate & 3.9 & $0.7 / 2.3$ \\
\hline
\end{tabular}

implemented in both JCMD scheme with and without rotation. That means the performance gain of JCMD does not exactly come from the interleavers compared with BICM scheme. We can still obtain significant gain through the optimal rotation and the matching of channel coding and labeling. Furthermore, the $4 \times 4$ JCMD MIMO system with the reverse spatial Q interleaver can obtain about 0.6 $\mathrm{dB}$ SNR gain compared with that with the cyclic-shift spatial Q interleaver. The results coincide well with the above analysis in Section 3.

For the QPSK $4 \times 4$ MIMO system, the optimal angles for $1 / 2$ and $3 / 4$ rate are $18^{\circ}$ and $25^{\circ}$, respectively. As shown in Figure 13, JCMD systems with the optimal rotation angle can obtain 0.6 and $3.9 \mathrm{~dB}$ SNR gains compared with the conventional BICM systems employing the ideal SVD precoding for low rate $1 / 2$ and high rate $3 / 4$, respectively. Given the optimal rotation angle, JCMD-MIMO with the reverse interleaver obtains significant 0.3 and $1.3 \mathrm{~dB}$ SNR gains at $\mathrm{BER}=10^{-5}$ compared to that with the cyclicshift interleaver for the code rate $1 / 2$ and $3 / 4$, respectively. Meanwhile, the proposed $1 / 2$ rate JCMD system with the reverse interleaver is only about $1.1 \mathrm{~dB}$ SNR gap to the JCMD limit for $4 \times 4$ MIMO. For the $3 / 4$ rate JCMD MIMO, the gap to the JCMD limit is reduced to $0.7 \mathrm{~dB}$ for $4 \times 4$ MIMO. SNR gains and gaps are summarized in Table 1. From the results, SNR gain becomes bigger for higher code rate.

\subsection{Results of JCMD-ID system on fast fading channels}

For the JCMD-ID 16QAM MIMO system, the optimal rotation angle is obtained by maximizing CM-AMI in Figure 9 , and it is $45^{\circ}$ at $1 / 2$ rate for $4 \times 4$ MIMO systems. In order to confirm our optimization method for JCMD-ID MIMO system, simulations are carried out with proposed $[63,32]_{8}$ BCC-coded JCMD-ID scheme on fast fading channels for $4 \times 4$ MIMO systems. The size of coded block $N=64,800$ bits and the maximal global iterative number is 30 . The powerful $1 / 2$ rate DVB-T2 LDPC-coded Gray-labeling BICM and BICM-ID schemes with the same block size are also simulated as the reference, and the same ideal SVD precoding method is used for the conventional BICM and BICM-ID schemes and the proposed JCMD-ID scheme.

The BER performance comparisons are shown in Figure 14.

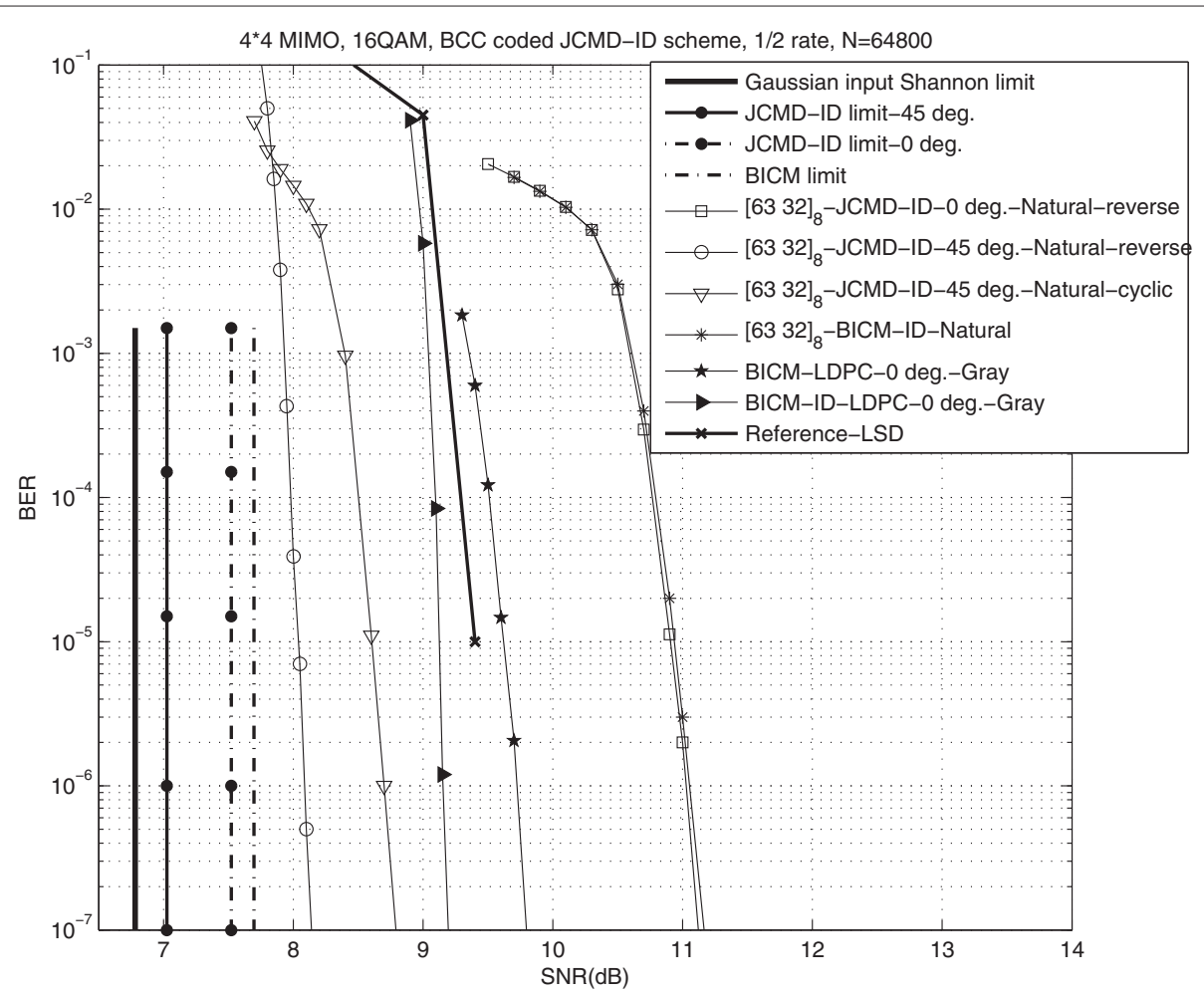

Figure 14 BER performance of rate-1/2, 16QAM $4 \times 4$ JCMD-ID MIMO systems on fast fading channels. 
Table 2 Average decoding complexity for each information bit

\begin{tabular}{llll}
\hline Operation & Optimized BCC & Turbo used in [24] & DVB-T2 LDPC \\
\hline Additions & 331 & 816 & 997.5 \\
Max ops. & 158 & 288 & 0 \\
Look-ups & 0 & 0 & 735 \\
\hline
\end{tabular}

For the $4 \times 4$ MIMO system with natural labeling, the optimized BCC $[63,32]_{8}$ coded JCMD-ID scheme with $45^{\circ}$ rotation and reverse $\mathrm{Q}$ interleaver exhibits excellent performance, which is only 1.3 and $1.1 \mathrm{~dB}$ away from the Gaussian-input capacity and JCMD-ID limit, respectively. It can obtain about $2.9 \mathrm{~dB}$ SNR gain compared with the BCC-coded BICM-ID scheme and JCMD-ID without rotation, which coincides with the above EXIT analysis. In addition, the scheme with the reverse interleaver obtains $0.4 \mathrm{~dB}$ SNR gain at $\mathrm{BER}=10^{-5}$ compared with that with cyclic-shift Q interleaver, which also proves the above analysis. Furthermore, the optimized JCMDID scheme also outperforms the DVB-T2 LDPC-coded BICM-ID scheme and the turbo-coded BICM-ID scheme in [24] by 0.9 and $1.4 \mathrm{~dB}$ gains, respectively.

For the JCMD-ID and conventional BICM-ID schemes, the main complexity lies in the channel decoding. The Max-Log-MAP algorithm is a simplified algorithm for the decoding of turbo code and BCC. For each decoding iteration, it requires $10 \times 2^{N_{\operatorname{Reg}}}+11$ additions and $5 \times 2^{N_{\operatorname{Reg}}}-2$ maximum operations [33]. The BCC decoding only needs one iteration (one Max-Log-MAP operation), while turbo decoding needs eight iterations (16 Max-Log-MAP operations). For the Log-BP algorithm of LDPC decoding, each iteration requires $M d_{c}\left(d_{c}-1\right)+N d_{v}\left(d_{v}-1\right)+N d_{c}$ additions and $M d_{c}^{2}$ look-up table operations, where $M$ is the number of the parity bits, and $d_{c}$ and $d_{v}$ are the average degree distributions of check nodes and variable nodes, respectively [34]. LDPC decoding requires 30 iterations. Thus, we can obtain the average decoding complexity comparison for each information bit, as shown in Table 2. Assuming the equal complexity of the three operations (addition, max, look-up), the decoding complexity of BCC is just is $44.3 \%$ and $28.2 \%$ of turbo and LDPC decoding, respectively. Therefore, the optimized BCC has much lower complexity compared with the DVB-T2 LDPC and turbo codes. This indicates that the optimized scheme with BCC coding obtains much better performance with much lower complexity.

\section{Conclusions}

A high-spectral-efficient JCMD scheme over MIMO fading channels is proposed. By jointly optimizing the component interleaver, the rotation modulation, and the $\mathrm{BCC}$ code, this scheme exhibits excellent performance. An optimum spatial component interleaver is proposed to maximize the achievable rate. For real-valued signals, we prove that the achievable rate of JCMD MIMO is greater than that of the conventional BICM MIMO scheme and $\frac{\pi}{4}$ is the optimal rotation angle. For the rotated QAM, the optimal rotation angles are investigated for the MIMO system according to the maximizing AMI criterion. For the JCMD-ID MIMO system, a simple GA-based search algorithm of $\mathrm{BCC}$ generator polynomials is also proposed to match the rotation modulation. Simulation results prove that this new scheme can significantly outperform the conventional turbo-coded BICM-ID scheme over MIMO fading channels by $1.4 \mathrm{~dB}$ SNR gain, while it has much lower complexity. In a word, the proposed JCMD scheme is simple, efficient, and robust for the future wireless communication systems.

\section{Competing interests}

The authors declare that they have no competing interests.

\section{Acknowledgements}

This work is sponsored by the National Natural Science Fund (61171101), National Great Science Specific Project (2009ZX03003-011-03) of People's Republic of China and 2014 Doctorial Innovation Fund of BUPT (CX201426) and the Fundamental Research Funds for the Central Universities.

Received: 23 July 2014 Accepted: 16 February 2015

Published online: 24 March 2015

\section{References}

1. C Felita, M Suryanegara, in Proceedings of International Conference on QiR (Quality in Research): 25-28 June 2013; Yogyakarta. 5 g key technologies: identifying innovation opportunity (IEEE, Piscataway, 2013), pp. 235-238

2. G Caire, G Taricco, E Biglieri, Bit-interleaved coded modulation. IEEE Trans. Inf. Theory. 44(3), 927-946 (1998)

3. X Li, JA Ritcey, Bit-interleaved coded modulation with iterative decoding. IEEE Commun. Lett. 1(6), 169-171 (1997)

4. B Vucetic, J Yuan, Space-Time Coding. (John Wiley \& Sons, Inc., England, 2003)

5. G Foschini, Layered space-time architecture for wireless communication in a fading environment when using multi-element antennas. Bell Labs Tech. J. 1(2), 41-59 (1996)

6. HE Gamal, AR Hammons, The layered space-time architecture: a new perspective. IEEE Trans. Inf. Theory. 1, 2321-2334 (2001)

7. J Boutros, E Viterbo, Signal space diversity: a power and bandwidth efficient diversity technique for the Rayleigh fading channel. IEEE Trans. Inf. Theory. 44(4), 1453-1467 (1998)

8. CA Nour, C Douillard, in Proceedings of 5 th International Symposium on Turbo Codes and Related Topics: 1-5 Sept. 2008; Lausanne. Improving BICM performance of QAM constellations for broadcasting applications (IEEE, Piscataway, 2008), pp. 55-60

9. NF Kiyani, UH Rizvi, JH Weber, GJM Janssen, in Proceedings of IEEE Wireless Communications and Networking Conference: 11-15 March 2007; Kowloon. Optimized rotations for LDPC-coded MPSK constellations with signal space diversity (IEEE, Piscataway, 2007), pp. 677-681

10. NF Kiyani, JH Weber, in Proceedings of IEEE Symposium on Communications and Vehicular Technology in the Benelux: 15-15 Nov. 2007; Delft. OFDM with BICM-ID and rotated MPSK constellations and signal space diversity (IEEE, Piscataway, NJ, USA, 2007), pp. 1-4

11. NF Kiyani, JH Weber, Exit chart analysis of iterative demodulation and decoding of MPSK constellations with signal space diversity. J. Commun. 3(3), 43-50 (2008)

12. NH Tran, HH Nguyen, T Le-Ngoc, Performance of BICM-ID with signal space diversity. IEEE Trans. Wireless Commun. 6(5), 1732-1742 (2007)

13. M Zhenzhou, S Zhiping, Z Chong, Z Zhongpei, in Proceedings of International Conference on Communications, Circuits and Systems: 25-27 
May 2008; Fujian. Design of signal space diversity based on non-binary LDPC code (IEEE, Piscataway, 2008), pp. 31-34

14. W Zhanji, W Wenbo, Improved coding-rotated-modulation orthogonal frequency division multiplexing system. IET Commun. 6(3), 272-280 (2012)

15. W Zhanji, W Wenbo, in Proceedings of 2010 Global Mobile Congress: 18-19 Oct. 2010; Shanghai. A novel joint-coding-modulation-diversity OFDM system (IEEE, Piscataway, 2010), pp. 1-6

16. X Qiuliang, S Jian, P Kewu, Y Fang, W Zhaocheng, Coded modulation with signal space diversity. IEEE Trans. Wireless Commun. 10(2), 660-668 (2011)

17. N Sharma, CB Papadias, Improved quasi-orthogonal codes through constellation rotation. IEEE Trans. Wireless Commun. 51(3), 332-335 (2003)

18. W An Zhong, Z Jian Kang, in Proceedings of 11th Canadian Workshop on Information Theory (CWIT): 13-15 May 2009; Ottawa. Novel rotation angle for quasi-orthogonal space-time block codes (IEEE, Piscataway, 2009), pp. $213-216$

19. W Su, X-G Xia, Signal constellations for quasi-orthogonal space time block codes with full diversity. IEEE Trans. Inf. Theory. 50(10), 2331-2347 (2004)

20. D Dung Ngoc, C Tellambura, in Proceedings of IEEE Global Telecommunications Conference (GLOBECOM): Dec. 2005; St. Louis. Optimal rotations for quasi-orthogonal STBC with two-dimensional constellations (IEEE, Piscataway, 2005), pp. 2317-2321

21. L Yueqian, M Salehi, in Proceedings of 46th Annual Conference on Information Sciences and Systems (CISS): 21-23 March 2012; Princeton. Coded MIMO systems with modulation diversity for block-fading channels (IEEE, Piscataway, 2012), pp. 1-5

22. H Lee, A Paulraj, MIMO systems based on modulation diversity. IEEE Trans. Wireless Commun. 58(12), 3045-3049 (2010)

23. H Soon Up, C Jinyong, J Sungho, R Ho Jin, S Jongsoo, in Proceedings of IEEE International Symposium on Broadband Multimedia Systems and Broadcasting: 13-15 May 2009; Bilbao. Performance evaluation of MIMO-OFDM with signal space diversity over frequency selective channels (IEEE, Piscataway, NJ, USA, 2009), pp. 1-5

24. BM Hochwald, S ten Brink, Achieving near-capacity on a multiple-antenna channel. IEEE Trans. Commun. 51(3), 389-399 (2003)

25. SY Goff, Signal constellation for bit-interleaved coded modulation. IEEE Trans. Inf. Theory. 49(1), 307-313 (2003)

26. FM Gardner, JD Baker, Simulation Techniques: Models of Communication Signal and Processes. (Wiley, New York, 1995)

27. S ten Brink, Convergence behavior of iteratively decoded parallel concatenated codes. IEEE Transa. Commun. 49(10), 1727-1737 (2001)

28. S ten Brink, Designing iterative decoding schemes with the extrinsic information transfer chart. AEU Int. J. Electron. Commun. 54(6), 389-398 (2000)

29. A Ashikhmin, G Kramer, S ten Brink, Extrinsic information transfer functions: model and erasure channel properties. IEEE Trans. Inf. Theory. 50(11), 2657-2673 (2004)

30. S Pfletschinger, F Sanzi, Error floor removal for bit-interleaved coded modulation with iterative detection. IEEE Trans. Wireless Commun. 5(11), 3174-3181 (2006)

31. P Guo, W Xuezhi, H Yingshi, in Proceedings of 3rd International Conference on Biomedical Engineering and Informatics: 16-18 Oct. 2010; Yantai. The enhanced genetic algorithms for the optimization design (IEEE, Piscataway, 2010), pp. 2990-2994

32. Frame structure channel coding and modulation for a second generation digital terrestrial television broadcasting system (DVB-T2). ETSI Std. DVB Document A122 [S] (June 2008)

33. P Robertson, E Villebrun, $\mathrm{P}$ Hoeher, in Proceedings of IEEE International Conference on Communications: 18-22 Jun 1995; Seattle. A comparison of optimal and sub-optimal map decoding algorithms operating in the log domain, (1995), pp. 1009-1013

34. C Jinghu, A Dholakia, E Eleftheriou, MPC Fossorier, Reduced-complexity decoding of LDPC codes. IEEE Trans. Commun. 53(8), 1288-1299 (2005)

\section{Submit your manuscript to a SpringerOpen ${ }^{\circ}$ journal and benefit from:}

- Convenient online submission

- Rigorous peer review

- Immediate publication on acceptance

- Open access: articles freely available online

- High visibility within the field

- Retaining the copyright to your article

Submit your next manuscript at $\boldsymbol{\wedge}$ springeropen.com 